\title{
Indirect searches of Galactic diffuse dark matter in INO-MagICAL detector
}

\author{
Amina Khatun, ${ }^{a, b}$ Ranjan Laha $^{c, d}$ and Sanjib Kumar Agarwalla ${ }^{a, b}$ \\ ${ }^{a}$ Institute of Physics, Sachivalaya Marg, \\ Sainik School Post, Bhubaneswar 751005, India \\ ${ }^{b}$ Homi Bhabha National Institute, Training School Complex, \\ Anushakti Nagar, Mumbai 400085, India \\ ${ }^{c}$ Kavli Institute for Particle Astrophysics and Cosmology (KIPAC), \\ Department of Physics, Stanford University, \\ Stanford, CA 94305, U.S.A. \\ ${ }^{d}$ SLAC National Accelerator Laboratory, \\ Menlo Park, CA 94025, U.S.A. \\ E-mail: amina@iopb.res.in, rlaha@stanford.edu, sanjib@iopb.res.in
}

ABSTRACT: The signatures for the existence of dark matter are revealed only through its gravitational interaction. Theoretical arguments support that the Weakly Interacting Massive Particle (WIMP) can be a class of dark matter and it can annihilate and/or decay to Standard Model particles, among which neutrino is a favorable candidate. We show that the proposed $50 \mathrm{kt}$ Magnetized Iron CALorimeter (MagICAL) detector under the India-based Neutrino Observatory (INO) project can play an important role in the indirect searches of Galactic diffuse dark matter in the neutrino and antineutrino mode separately. We present the sensitivity of $500 \mathrm{kt} \cdot \mathrm{yr}$ MagICAL detector to set limits on the velocityaveraged self-annihilation cross-section $(\langle\sigma v\rangle)$ and decay lifetime $(\tau)$ of dark matter having mass in the range of $2 \mathrm{GeV} \leq m_{\chi} \leq 90 \mathrm{GeV}$ and $4 \mathrm{GeV} \leq m_{\chi} \leq 180 \mathrm{GeV}$ respectively, assuming no excess over the conventional atmospheric neutrino and antineutrino fluxes at the INO site. Our limits for low mass dark matter constrain the parameter space which has not been explored before. We show that MagICAL will be able to set competitive constraints, $\langle\sigma v\rangle \leq 1.87 \times 10^{-24} \mathrm{~cm}^{3} \mathrm{~s}^{-1}$ for $\chi \chi \rightarrow \nu \bar{\nu}$ and $\tau \geq 4.8 \times 10^{24} \mathrm{~s}$ for $\chi \rightarrow \nu \bar{\nu}$ at $90 \%$ C.L. (1 d.o.f.) for $m_{\chi}=10 \mathrm{GeV}$ assuming the NFW as dark matter density profile.

Keywords: Dark matter, Neutrino Detectors and Telescopes (experiments), Beyond Standard Model

ArXiv EPrint: 1703.10221 


\section{Contents}

1 Introduction and motivation $\quad 1$

2 Discussions on dark matter 3

2.1 Dark matter density profile 3

2.2 Annihilation of dark matter 4

2.3 Decay of dark matter 5

$\begin{array}{lll}3 & \text { Key features of ICAL detector } & 7\end{array}$

$\begin{array}{llr}4 & \text { Event spectrum and rates } & 8\end{array}$

5 Simulation method 10

6 Results 11

6.1 Constraints on annihilation of dark matter 11

$\begin{array}{lll}6.2 & \text { Constraints on decay of dark matter } & 14\end{array}$

$\begin{array}{ll}\text { 6.3 Comparison with other experiments } & 16\end{array}$

$\begin{array}{ll}\text { 6.4 The constraints on DM-induced neutrino flux } & 18\end{array}$

$\begin{array}{lll}7 & \text { Conclusions } & 19\end{array}$

$\begin{array}{ll}\text { A Oscillation of DM induced neutrinos } & 20\end{array}$

\section{Introduction and motivation}

Plethora of attempts are being made in the intensity, energy, and cosmic frontiers to build up knowledge about the Universe. Recent observations by Planck satellite [1] confirm that the baryonic and unknown non-baryonic matter (dark matter) contribute $\sim 4.8 \%$ and $\sim 26 \%$ of the total energy density of the Universe respectively. The first indication for the existence of dark matter (DM) in the Universe was made by the Swiss astronomer Fritz Zwicky [2]. This observation was put on a solid footing by Vera Rubin and her collaborators [3]. The astrophysical [4, 5] and cosmological observations [6, 7] confirm the existence of dark matter from the length scales of a few kpc to a few Gpc.

All the astrophysical evidences of dark matter are through its gravitational interactions. The non-gravitational particle physics properties of DM particles are completely unknown. The relic abundance of cold dark matter (CDM) in the Universe is matched assuming a $\sim 100 \mathrm{GeV}$ dark matter particle with electro-weak coupling strength. This class of particles is known as Weakly Interacting Massive Particle (WIMP) [8-10]. Supersymmetry, one of 
the most favored beyond-the-Standard Model theory, also predicts more than one dark matter candidates including the WIMP [11].

There are three types of detection methods for the search of DM: (i) Direct detection: DM particles are detected by observing recoiled nuclei from the scattering of DM particles in the laboratory. Experiments such as DAMA/LIBRA [12], LUX [13], CDMS [14], XENON [15], DarkSide [16], and PandaX [17] pursue this strategy. (ii) Indirect detection: it is possible that DM particles can decay and/or annihilate to any of the Standard Model (SM) particles like $\nu \bar{\nu}, t \bar{t}, b \bar{b}$ etc. An excess (over standard astrophysical backgrounds) of these SM particles can be searched for to understand dark matter. The unstable SM particles decay to produce neutrinos and photons which can be searched for indirect detection. The prospects of dark matter searches through neutrino portal has been studied in the literature [18-32]. Fermi-LAT presents the analysis of its collected data of gamma rays having the energy in the range of $200 \mathrm{MeV}$ to $500 \mathrm{GeV}$ from Galactic halo in 5.8 years in ref. [33]. Multiwavelength searches for dark matter have complementary reach [34]. Our focus in this work is indirect detection of dark matter via neutrinos and antineutrinos. (iii) Collider searches: the searches for supersymmetric DM candidates are carried out in LHC [35-37].

The 50 kt Magnetized Iron CALorimeter (MagICAL ${ }^{1}$ ) detector is proposed to be built by the India-based Neutrino Observatory (INO) project to observe the atmospheric neutrino and antineutrino separately having energy in multi-GeV range and covering a wide ranges of path lengths (few $\mathrm{km}$ to few thousands of $\mathrm{km}$ ) through the Earth matter. The primary mission of the MagICAL detector is to unravel the mass ordering ${ }^{2}$ (MO) of neutrino using the Earth matter effect [38-41] and to measure the neutrino mixing parameters precisely [39, 42, 43]. The MagICAL detector has also the potential to explore the physics beyond the Standard Model [44-48]. In our study, we show that the MagICAL detector can play a very important role in the indirect search of DM having mass in the multi-GeV range with the help of its excellent detection efficiency, energy, and angular resolutions. We explore the sensitivity of the MagICAL detector to detect the neutrino and antineutrino events coming from the diffuse dark matter annihilation/decay in the Milky Way galaxy. We present the constraint on the self-annihilation cross-section $(\langle\sigma v\rangle)$ and the decay lifetime $(\tau)$ of diffuse dark matter having mass in the range $[2,90] \mathrm{GeV}$ and $[4,180] \mathrm{GeV}$ respectively using $500 \mathrm{kt} \cdot \mathrm{yr}$ exposure of the MagICAL detector.

We describe the dark matter density profile and the calculation of annihilation and decay rate of dark matter in section 2. The key features of the MagICAL detector is presented in section 3. Section 4 deals with the expected event distribution of atmospheric and DM induced neutrinos in the MagICAL detector. We present the simulation method in section 5. The prospective limits on the self-annihilation cross-section and decay lifetime of dark matter are presented in section 6 . We compare our results with the existing bounds

\footnotetext{
${ }^{1}$ The "MagICAL" name is used here as the abbreviation of Magnetized Iron CALorimeter which is commonly known as ICAL detector. We prefer the name MagICAL to emphasize that magnetic field is present in the ICAL detector, which enable us to separate neutrino and anti-neutrino events.

${ }^{2}$ Two distinct patterns of neutrino masses are allowed: $m_{3}^{2}>m_{2}^{2}>m_{1}^{2}$, known as normal ordering (NO) where $\Delta m_{31}^{2}\left(\equiv m_{3}^{2}-m_{1}^{2}\right)>0$ and $m_{2}^{2}>m_{1}^{2}>m_{3}^{2}$, called inverted ordering (IO) where $\Delta m_{31}^{2}<0$.
} 


\begin{tabular}{|c|c|c|c|}
\hline & $(\alpha, \beta, \gamma, \delta)$ & $\rho_{\mathrm{sc}}\left[\mathrm{GeV} \mathrm{cm}^{-3}\right]$ & $r_{s}[\mathrm{kpc}]$ \\
\hline NFW & $(1,3,1,0)$ & 0.471 & 16.1 \\
Burkert & $(2,3,1,1)$ & 0.487 & 9.26 \\
\hline
\end{tabular}

Table 1. The value of parameters associated with the NFW and Burkert profiles are listed here. They are same as in ref. [65].

from other experiments. We also study the flux upper limit due to dark matter induced neutrinos in the MagICAL detector. We conclude in section 7.

\section{Discussions on dark matter}

\subsection{Dark matter density profile}

The general parameterization of a spherically symmetric dark matter density profile is given by

$$
\rho(r)=\frac{\rho_{0}}{\left[\delta+r / r_{s}\right]^{\gamma} \cdot\left[1+\left(r / r_{s}\right)^{\alpha}\right]^{(\beta-\gamma) / \alpha}} .
$$

The density, $\rho(r)$, is expressed in $\mathrm{GeV} \mathrm{cm}^{-3}$ and $r$ is the distance from the center of the galaxy in kpc. The parameter, $r_{s}$, is the scale radius in kpc. The shape of the outer profile is controlled by $\alpha$ and $\beta$, whereas $\gamma$ parametrizes the slope of the inner profile. The dark matter density at the Solar radius $\left(\mathrm{R}_{\mathrm{sc}}\right)$ is denoted by $\rho_{\mathrm{sc}}$. We assume $\mathrm{R}_{\mathrm{sc}}=$ $8.5 \mathrm{kpc}$ [49]. The normalization constant, $\rho_{0}$, and all the results are calculated using the values of parameters as given in table 1.

Numerical simulations which involve only dark matter particles predict a cuspy profile [50-53]. Although these simulations reproduce the large-scale structure of the Universe, yet this prescription has challenges at scales below the size of a typical galaxy. It has been proposed that the addition of baryons can solve all of these small scale issues, although the results vary [54-62]. Present observations are not yet precise enough to distinguish between a cored and a cuspy profile [63].

To take this DM halo uncertainty into account, we generate all the results with two different DM profiles: Navarro Frenk White (NFW) profile [50], which represents cuspy halos, and the Burkert profile [64], which represents cored halos. The values of different parameters associated with these profiles are taken from ref. [65]. In figure 1(a), we plot the NFW and Burkert dark matter density profiles with distance $r$ from the center of the Milky Way galaxy by the black solid and green dashed lines respectively.

For conservativeness, we do not consider the effects of dark matter substructure. Depending on the value of the minimum halo mass and other astrophysical uncertainties, this can give a substantial contribution to the signal discussed here [66-71].

In figure 1(b), a schematic diagram of a small portion of the Milky Way DM halo is shown with $\mathrm{O}$ as the Galactic center (GC). The dark matter density at point $\mathrm{P}$ with its distance $l$ from the Earth is a function of the length $\mathrm{OP}=\sqrt{R_{\mathrm{sc}}^{2}-2 l R_{\mathrm{sc}} \cos \psi+l^{2}}$. The angle made at the Earth by points $\mathrm{P}$ and $\mathrm{O}$ is $\psi$ and the corresponding solid angle is $\Delta \Omega$. 


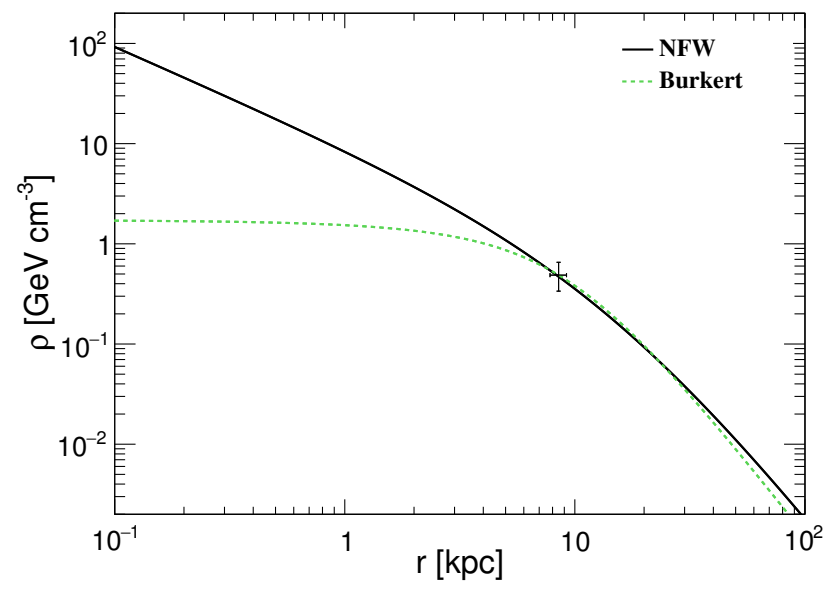

(a)

$$
\begin{gathered}
\mathrm{O}=\text { Galactic Center } \\
\mathrm{OP}=\sqrt{R_{s c}^{2}-2 l R_{s c} \cos \psi+l^{2}}
\end{gathered}
$$

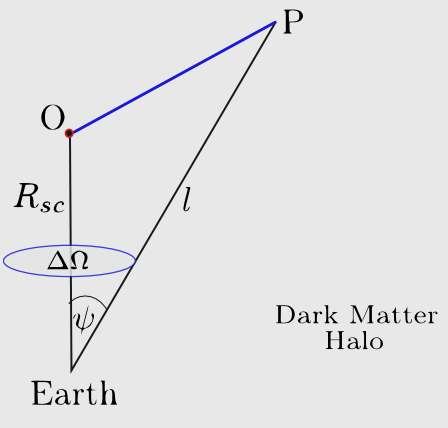

(b)

Figure 1. (a) Distribution of the dark matter density in the Milky Way galaxy for the NFW (black solid line) and Burkert profiles (green dashed line). The observational bounds on local dark matter density $\left(\rho_{\mathrm{sc}}\right)$ and the solar radius $\left(R_{\mathrm{sc}}\right)$ and their $2 \sigma$ uncertainties are indicated $[4,65] .(\mathrm{b})$ A schematic diagram of some part of the Milky Way dark matter halo. The Galactic center (GC) is denoted by $\mathrm{O}$ and $R_{\mathrm{sc}}$ is the distance between the Earth and the GC. The parameter $l$ is the distance between point $\mathrm{P}$ and the Earth. The angle made at the Earth by points $\mathrm{P}$ and $\mathrm{O}$ and the corresponding solid angle are denoted by $\psi$ and $\Delta \Omega$ respectively.

\subsection{Annihilation of dark matter}

We consider the annihilation between a dark matter particle $(\chi)$ and its antiparticle $(\bar{\chi})$ to produce a neutrino and an antineutrino in the final state with $100 \%$ branching ratio:

$$
\chi+\bar{\chi} \rightarrow \nu+\bar{\nu} .
$$

The neutrinos and antineutrinos of $e, \mu$, and $\tau$ flavors are assumed to be produced in 1:1:1 ratio at source. This ratio remains same on arrival at the Earth surface due to loss of coherence while propagating through astrophysical distances (see appendix A).

The number of $\nu / \bar{\nu}$ from a direction $\psi$ due to the annihilation of dark matter particles is proportional to the line of sight integration of the square of dark matter density:

$$
\mathcal{J}^{\mathrm{ann}}(\psi)=\frac{1}{R_{\mathrm{sc}} \rho_{\mathrm{sc}}^{2}} \int_{0}^{l_{\mathrm{max}}} d l \rho^{2}\left(\sqrt{R_{\mathrm{sc}}^{2}-2 l R_{\mathrm{sc}} \cos \psi+l^{2}}\right) .
$$

The factor $\frac{1}{R_{\mathrm{sc}} \rho_{\mathrm{sc}}^{2}}$ is included to make $\mathcal{J}^{\mathrm{ann}}(\psi)$ dimensionless. The upper limit $l_{\max }$ is the distance between the observer and the farthest point (denoted by $\mathrm{P}^{\prime}$ ) in the Milky Way halo at the angle $\psi$. The radius of Milky Way galaxy is $R_{\mathrm{MW}}\left(=\mathrm{OP}^{\prime}=100 \mathrm{kpc}\right)$, and thus

$$
l_{\max }=\sqrt{\left(R_{\mathrm{MW}}^{2}-R_{\mathrm{sc}}^{2} \sin ^{2} \psi\right)}+R_{\mathrm{sc}} \cos \psi .
$$

Increase of $R_{\mathrm{MW}}$ to $150 \mathrm{kpc}$ enhances the value of $\mathcal{J}^{\mathrm{ann}}\left(\psi=180^{\circ}\right)$ by $0.03 \%$. The average value of $\mathcal{J}^{\text {ann }}(\psi)$ over a solid angle $2 \pi \int_{0}^{\psi} \sin \psi^{\prime} d \psi^{\prime}=2 \pi(1-\cos \psi)$ is

$$
\mathcal{J}_{\Delta \Omega}^{\mathrm{ann}}(\psi)=\frac{1}{2 \pi(1-\cos \psi)} \int_{\cos \psi}^{1} 2 \pi d\left(\cos \psi^{\prime}\right) \mathcal{J}^{\mathrm{ann}}\left(\psi^{\prime}\right) .
$$



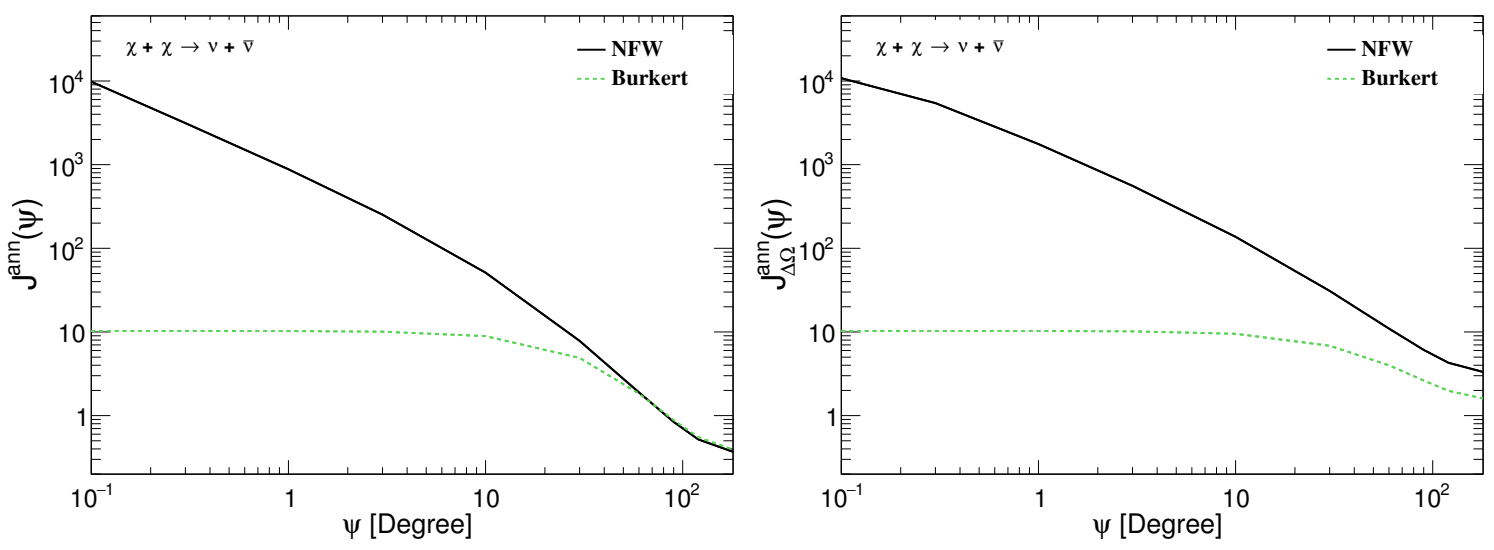

Figure 2. The value of $\mathcal{J}^{\text {ann }}(\psi)$ (see eq. (2.3)) and its average $\left(\mathcal{J}_{\Delta \Omega}^{\operatorname{ann}}(\psi)\right)$ over solid angle $\Delta \Omega$ $=2 \pi(1-\cos \psi)$ (see eq. (2.5)) are shown in left and right panels. In both the panels, black solid and green dashed lines present the corresponding quantities for the NFW and Burkert profiles respectively. We use $\mathcal{J}_{\Delta \Omega}^{\operatorname{ann}}\left(\psi=180^{\circ}\right)$ for the diffuse dark matter analysis, which has values 3.33 and 1.6 for the NFW and Burkert profiles respectively.

The variation of $\mathcal{J}^{\text {ann }}(\psi)$ and $\mathcal{J}_{\Delta \Omega}^{\text {ann }}(\psi)$ with angle $\psi$ are shown by the black solid (green dashed) lines in left and right panels of figure 2 respectively using the NFW (Burkert) DM halo profile. The value of $\mathcal{J}_{\Delta \Omega}^{\text {ann }}=3.33$ for the NFW profile and $\mathcal{J}_{\Delta \Omega}^{\text {ann }}=1.6$ for the Burkert profile with $\Delta \Omega=4 \pi$. The flux of each flavor of $\nu / \bar{\nu}$ per unit energy range per unit solid angle (in units of $\mathrm{GeV}^{-1} \mathrm{sr}^{-1} \mathrm{~cm}^{-2} \mathrm{~s}^{-1}$ ) produced in the final state of dark matter particles annihilation is given by

$$
\frac{d^{2} \Phi_{\nu / \bar{\nu}}^{\mathrm{ann}}}{d E d \Omega}=\frac{\left\langle\sigma_{A} v\right\rangle}{2} \mathcal{J}_{\Delta \Omega}^{\mathrm{ann}} \frac{R_{\mathrm{sc}} \rho_{\mathrm{sc}}^{2}}{4 \pi m_{\chi}^{2}} \frac{1}{3} \frac{d N^{\mathrm{ann}}}{d E},
$$

where $\left\langle\sigma_{A} v\right\rangle$ is the self-annihilation cross-section in units of $\mathrm{cm}^{3} \mathrm{~s}^{-1}$. The factor $\frac{1}{2}$ is included as we assume the dark matter particle is same as its own antiparticle. The factor $\frac{1}{3}$ takes into account the flavor ratio of $\nu / \bar{\nu}$ on the Earth's surface. The probability of $\nu_{e}$, $\nu_{\mu}$, and $\nu_{\tau}$ to be produced in the final state are the same. Therefore the flux of $\nu / \bar{\nu}$ with each lepton flavor is calculated as the total $\nu / \bar{\nu}$ flux divided by the total number of lepton generations, which gives rise to the $\frac{1}{3}$ factor in eq. (2.6). The factor $4 \pi$ in the denominator is for the isotropic production of $\nu \bar{\nu}$ in annihilation of dark matter. The parameter $m_{\chi}$ is mass of the DM particles in units of GeV. The energy spectrum of $\nu / \bar{\nu}$ is given by

$$
\frac{d N^{\mathrm{ann}}}{d E}=\delta\left(E_{\nu / \bar{\nu}}-m_{\chi}\right),
$$

since dark matter particles in our galaxy are non-relativistic (local velocity $\sim 10^{-3} \mathrm{c}$ ).

\subsection{Decay of dark matter}

A dark matter particle is assumed to decay into $\nu_{e}+\bar{\nu}_{e}, \nu_{\mu}+\bar{\nu}_{\mu}$, and $\nu_{\tau}+\bar{\nu}_{\tau}$ with equal branching ratio:

$$
\chi \rightarrow \nu+\bar{\nu} .
$$



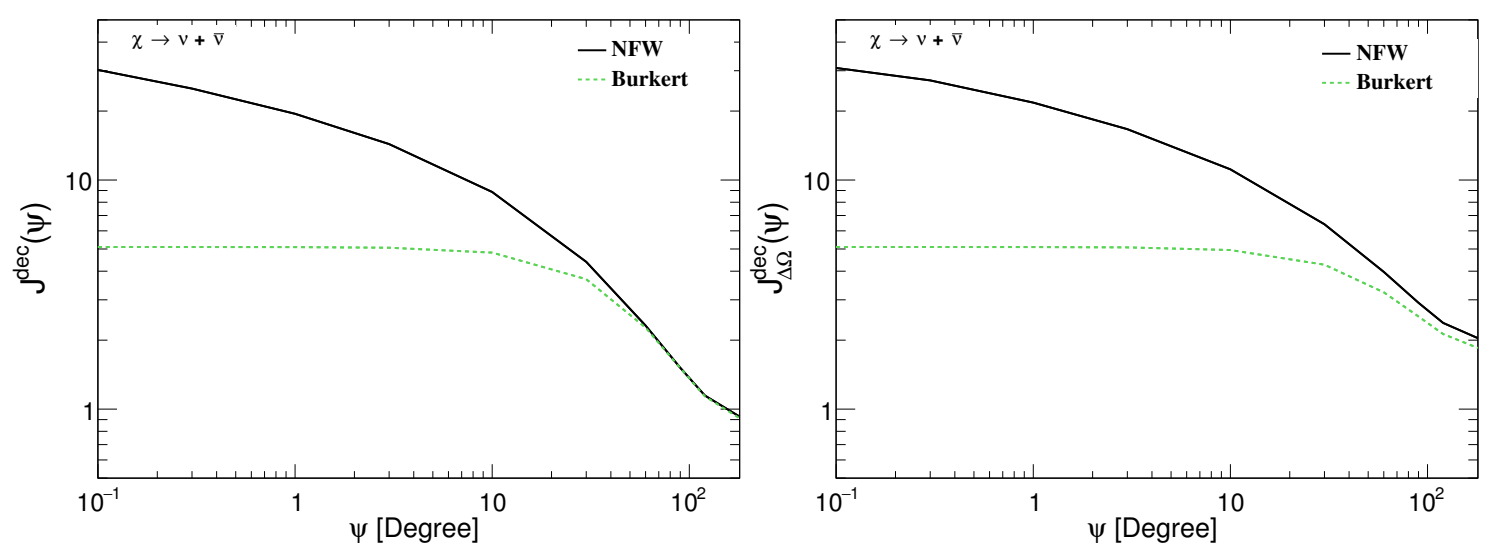

Figure 3. Line of sight integral for dark matter decay, $\mathcal{J}^{\operatorname{dec}}(\psi)$, (see eq. (2.9)) vs. $\psi$ and the average value of $\mathcal{J}^{\operatorname{dec}}(\psi)$ over solid angle $\Delta \Omega$, i.e., $\mathcal{J}_{\Delta \Omega}^{\text {dec }}(\psi)$ (see eq. (2.10)) for the decay process are shown in left and right panels respectively. In both the panels black solid and green dashed lines present the corresponding quantities for the NFW and the Burkert profiles respectively. We use the value of $\mathcal{J}_{\Delta \Omega}^{\text {dec }}\left(\psi=180^{\circ}\right)$ in our analysis, which are given by 2.04 and 1.85 for the NFW and Burkert profiles respectively.

The $\nu / \bar{\nu}$ flux from dark matter decay is proportional to the line of sight integral of the dark matter distribution, $\mathcal{J}^{\text {dec }}(\psi)$, with

$$
\mathcal{J}^{\mathrm{dec}}(\psi)=\frac{1}{R_{\mathrm{sc}} \rho_{\mathrm{sc}}} \int_{0}^{l_{\mathrm{max}}} d l \rho\left(\sqrt{R_{\mathrm{sc}}^{2}-2 l R_{\mathrm{sc}} \cos \psi+l^{2}}\right) .
$$

The quantity $R_{\mathrm{sc}} \rho_{\mathrm{sc}}$ in the denominator makes $\mathcal{J}^{\operatorname{dec}}(\psi)$ dimensionless. All other symbols have same meaning as before. The quantity $\mathcal{J}_{\Delta \Omega}^{\text {dec }}(\psi)$ represents the average value of $\mathcal{J}^{\text {dec }}(\psi)$ over the solid angle $\Delta \Omega=2 \pi(1-\cos \psi)$ :

$$
\mathcal{J}_{\Delta \Omega}^{\mathrm{dec}}(\psi)=\frac{1}{2 \pi(1-\cos \psi)} \int_{\cos \psi}^{1} 2 \pi d\left(\cos \psi^{\prime}\right) \mathcal{J}^{\mathrm{dec}}\left(\psi^{\prime}\right)
$$

For the decaying dark matter, $\mathcal{J}^{\operatorname{dec}}(\psi)$ and $\mathcal{J}_{\Delta \Omega}^{\operatorname{dec}}(\psi)$ are shown in left and right panels of figure 3 respectively by the black solid (green dashed) lines using the NFW (Burkert) profile. We obtain $\mathcal{J}_{\Delta \Omega}^{\text {dec }}\left(\psi=180^{\circ}\right)=2.04$ and 1.85 for the NFW and Burkert profile respectively. These agree with those presented in ref. [72] up to uncertainties in the dark matter profile parameters.

The flux of neutrinos of each flavor per unit energy per unit solid angle in units of $\mathrm{GeV}^{-1} \mathrm{sr}^{-1} \mathrm{~cm}^{-2} \mathrm{~s}^{-1}$ from the decay of dark matter particles is given by

$$
\frac{d^{2} \Phi_{\nu / \bar{\nu}}^{\mathrm{dec}}}{d E d \Omega}=\mathcal{J}_{\Delta \Omega}^{\mathrm{dec}} \frac{R_{\mathrm{sc}} \rho_{\mathrm{sc}}}{4 \pi m_{\chi} \tau} \frac{1}{3} \frac{d N^{\mathrm{dec}}}{d E},
$$

where $m_{\chi}$ is the mass of $\mathrm{DM}$ particle $(\chi)$ in $\mathrm{GeV}$, and $\tau$ is the decay lifetime of $\chi$ in second. The factor $\frac{1}{3}$ accounts for the averaging over total number of lepton flavors and $4 \pi$ implies 


\begin{tabular}{|l|l|}
\hline Energy resolution $\left(\sigma_{E}\right)(\mathrm{GeV})$ & $0.1 \times(E / \mathrm{GeV})$ \\
\hline Angular resolution $(\Delta \theta)$ & $10^{\circ}$ \\
\hline Detection efficiency $(\mathcal{E})$ & $80 \%$ \\
\hline CID efficiency $(\mathcal{C})$ & $90 \%$ \\
\hline
\end{tabular}

Table 2. The detector characteristics used in the simulations. We use the same detector properties for $\mu^{-}$and $\mu^{+}$events.

isotropic decay. The mass of dark matter is shared by final $\nu$ and $\bar{\nu}$, thus, their energy spectrum can be written as

$$
\frac{d N^{\mathrm{dec}}}{d E}=\delta\left(E_{\nu / \bar{\nu}}-m_{\chi} / 2\right)
$$

\section{Key features of ICAL detector}

The proposed $50 \mathrm{kt}$ MagICAL detector $[40,73]$ is designed to have 151 alternate layers of $5.6 \mathrm{~cm}$ thick iron plates (act as target mass) and glass Resistive Plate Chambers (RPCs, act as active detector elements). The plan is to have a modular structure for the detector with a dimension of $48 \mathrm{~m}(\mathrm{~L}) \times 16 \mathrm{~m}(\mathrm{~W}) \times 14.5 \mathrm{~m}(\mathrm{H})$, subdivided into 3 modules, each having a dimension of $16 \mathrm{~m} \times 16 \mathrm{~m} \times 14.5 \mathrm{~m}$. The field strength of the magnetized iron plates will be around $1.5 \mathrm{~T}$, with fields greater than $1 \mathrm{~T}$ over at least $85 \%$ of the detector volume [74]. Bending of charged particles in this magnetic field help us to identify the charges of $\mu^{-}$and $\mu^{+}$which are produced in the charged-current (CC) interactions of $\nu_{\mu}$ and $\bar{\nu}_{\mu}$ inside the detector. This magnetic field inside the detector is best suited to observe muons having energies in $\mathrm{GeV}$ range, measure their charges, and reconstruct their momentum with high precision [75]. The capabilities of ICAL to measure three flavor oscillation parameters based on the information coming from muon energy $\left(E_{\mu}\right)$ and direction $\left(\cos \theta_{\mu}\right)$ have already been explored in refs. $[38,42]$. Recently it has been demonstrated that the ICAL detector has ability to detect hadron ${ }^{3}$ showers and extract information about hadron energy from them $[76,77]$. The energy of hadron $\left(E_{\text {had }}^{\prime}=E_{\nu}-E_{\mu}\right)$ can be calibrated using number of hits in the detector due to hadron showers [76]. In [39], it has been shown that by adding the hadron energy information $\left(E_{\text {had }}^{\prime}\right)$ to the muon information $\left(E_{\mu}, \cos \theta_{\mu}\right)$ of each event the sensitivity of ICAL to the neutrino oscillation parameters can be greatly enhanced. In this phenomenological study, we explore the physics reach of MagICAL to see the signatures of Galactic diffuse dark matter through neutrino portal using the neutrino energy $\left(E_{\nu}\right)$ and zenith angle $\left(\cos \theta_{\nu}\right)$ as reconstructed variables. We consider reconstructed neutrino energy threshold to be $1 \mathrm{GeV}$ for both $\mu^{-}$and $\mu^{+}$events. The energy resolution of the MagICAL detector is expected to be quite good, and we assume that the neutrino energy will be reconstructed with a Gaussian energy resolution of $10 \%$ of $E / \mathrm{GeV}$ (see table 2). As far as the angular resolution is concerned, we use a constant

\footnotetext{
${ }^{3}$ These final state hadrons are produced along with the muons in CC deep-inelastic scattering processes in multi-GeV energies, and they carry vital information about the initial neutrino.
} 
angular resolution of $10^{\circ}$. For $\mu^{\mp}$ events, the constant detection efficiency is $80 \%$, and the constant charge identification (CID) efficiency is $90 \%$. The detector properties that we use in our simulation agree quite well with the detector characteristics that have been considered in the existing phenomenological studies related to the MagICAL detector. For example see refs. [78-81]. We have checked that the representative choices of energy and angular resolutions of $\nu_{\mu}$ and $\bar{\nu}_{\mu}$ that we consider in this work can produce similar results for oscillation studies as obtained by the INO simulation code using muon momentum as variable. In this work, we assume that the $50 \mathrm{kt}$ MagICAL detector will collect atmospheric neutrino data for 10 years giving rise to a total exposure of $500 \mathrm{kt} \cdot \mathrm{yr}$.

\section{Event spectrum and rates}

In this section, we present the expected event spectra and total event rates at the MagICAL detector. To estimate the number of expected $\mu^{-}$events ${ }^{4}$ from atmospheric $\nu_{\mu}$ and $\bar{\nu}_{\mu}{ }^{5}$ in the $i$-th energy bin and $j$-th zenith bin at the MagICAL detector, we use the following expression [82]

$$
\begin{aligned}
N_{i j}^{a t m}\left(\mu^{-}\right)= & 2 \pi N_{\mathrm{t}} \mathcal{T} \int_{E_{\min }^{i}}^{E_{\max }^{i}} d E^{\prime} \int_{\cos \theta_{\min }^{j}}^{\cos \theta_{\max }^{j}} d\left(\cos \theta^{\prime}\right) \int_{-1}^{1} d(\cos \theta) \int_{0}^{\infty} d E R\left(E, E^{\prime}\right) \\
\times & R\left(\theta, \theta^{\prime}\right)\left[\sigma_{\nu_{\mu}}^{C C}(E) \mathcal{E} \mathcal{C}\left\{\frac{d^{2} \Phi_{\nu_{\mu}}}{d \cos \theta d E} P_{\mu \mu}+\frac{d^{2} \Phi_{\nu_{e}}}{d \cos \theta d E} P_{e \mu}\right\}\right. \\
& \left.+\bar{\sigma}_{\nu_{\mu}}^{C C}(E) \overline{\mathcal{E}}(1-\overline{\mathcal{C}})\left\{\frac{d^{2} \bar{\Phi}_{\nu_{\mu}}}{d \cos \theta d E} \bar{P}_{\mu \mu}+\frac{d^{2} \bar{\Phi}_{\nu_{e}}}{d \cos \theta d E} \bar{P}_{e \mu}\right\}\right] .
\end{aligned}
$$

In the above equation, $\mathcal{T}$ is the total running time in second, and $N_{t}$ is the total number of target nucleons in the detector. The quantities $E\left(E^{\prime}\right)$ and $\theta\left(\theta^{\prime}\right)$ are the true (reconstructed) neutrino energy and zenith angle respectively. For $\mu^{-}\left(\mu^{+}\right)$events, $\sigma_{\nu_{\mu}}^{C C}\left(\bar{\sigma}_{\nu_{\mu}}^{C C}\right)$ is the total neutrino (antineutrino) per nucleon CC cross-section. These cross-sections have been taken from figure 9 of ref. [83]. We take the unoscillated atmospheric $\nu_{\mu}$ and $\nu_{e}$ fluxes estimated for the INO site in units of $\mathrm{m}^{-2} \mathrm{~s}^{-1} \mathrm{GeV}^{-1} \mathrm{sr}^{-1}$ from ref. [84]. ${ }^{6}$ The probability of a $\nu_{\mu}\left(\nu_{e}\right)$ to survive (appear) as $\nu_{\mu}$ is denoted by $P_{\mu \mu}\left(P_{e \mu}\right)$. The parameters $\mathcal{E}(\overline{\mathcal{E}})$ and $\mathcal{C}(\overline{\mathcal{C}})$ are the detection and charge identification efficiencies respectively for $\mu^{-}\left(\mu^{+}\right)$events. The quantities $R\left(E, E^{\prime}\right)$ and $R\left(\theta, \theta^{\prime}\right)$ are the Gaussian energy and angular resolution functions of the detector, which are expressed in the following way

$$
R\left(E, E^{\prime}\right)=\frac{1}{\sigma_{E} \sqrt{2 \pi}} \exp \left\{\frac{-\left(E^{\prime}-E\right)^{2}}{2 \sigma_{E}^{2}}\right\}
$$

and

$$
R\left(\theta, \theta^{\prime}\right)=\frac{1}{\sigma_{\theta} \sqrt{2 \pi}} \exp \left\{\frac{-\left(\cos \theta^{\prime}-\cos \theta\right)^{2}}{2 \sigma_{\theta}^{2}}\right\} .
$$

\footnotetext{
${ }^{4}$ The number of $\mu^{+}$events from atmospheric neutrinos can be estimated using eq. (4.1) by considering appropriate flux, oscillation probability, cross-section, and detector properties.

${ }^{5}$ Atmospheric muon antineutrino flux gives rise to $\mu^{+}$events in the detector, which can be misidentified as $\mu^{-}$events.

${ }^{6}$ Atmospheric $\nu$ fluxes for the INO site are in http://www.icrr.u-tokyo.ac.jp/ mhonda/.
} 


$\left.\begin{array}{|l|c|c|c|c|}\hline \text { Observables } & \text { Range } & \text { Width } & \multicolumn{2}{|c|}{\text { Total bins }} \\ \hline & 1,15 & 1 & 14 \\ \mathrm{E}_{\nu}(\mathrm{GeV}) & 15,25 & 2 & 5 & \\ & 25,50 & 5 & 5 & 29 \\ & 50,100 & 10 & 5\end{array}\right\}$

Table 3. The binning scheme adopted for the reconstructed $E_{\nu}$ and $\cos \theta_{\nu}$ for each muon polarity. The last column depicts the total number of bins considered for each observable.

The parameters $\sigma_{E}$ and $\sigma_{\theta}(\sin \theta \Delta \theta)$ denote the energy and angular resolutions as given in table 2 .

We can estimate the $\mu^{-}$events in the $i$-th energy bin and $j$-th angular bin from the dark matter induced neutrinos and anitneutrinos by making suitable changes in eq. (4.1) in the following fashion

$$
\begin{aligned}
& N_{i j}^{d m}\left(\mu^{-}\right)=2 \pi N_{\mathrm{t}} \mathcal{T} \int_{E_{\min }^{i}}^{E_{\max }^{i}} d E^{\prime} \int_{\cos \theta_{\min }^{j}}^{\cos \theta_{\max }^{j}} d\left(\cos \theta^{\prime}\right) \int_{-1}^{1} d(\cos \theta) \int_{0}^{\infty} d E R\left(E, E^{\prime}\right) R\left(\theta, \theta^{\prime}\right) \\
& \times \frac{d^{2} \Phi^{d m}}{d \cos \theta d E}\left[\sigma_{\nu_{\mu}}^{C C}(E) \mathcal{E} \mathcal{C}\left\{P_{e \mu}+P_{\mu \mu}+P_{\tau \mu}\right\}+\bar{\sigma}_{\nu_{\mu}}^{C C}(E) \overline{\mathcal{E}}(1-\overline{\mathcal{C}})\left\{\bar{P}_{e \mu}+\bar{P}_{\mu \mu}+\bar{P}_{\tau \mu}\right\}\right] . \quad(4.4)
\end{aligned}
$$

In case of dark matter annihilation and decay, we have fluxes of $\nu_{\tau}$ and $\bar{\nu}_{\tau}$ along with the fluxes of $\nu_{e}, \bar{\nu}_{e}, \nu_{\mu}$, and $\bar{\nu}_{\mu}$. The dark matter induced neutrino and antineutrino fluxes ${ }^{7}$ for each flavor are estimated using eqs. (2.6) and (2.11) for annihilation and decay processes respectively. In the above equation, the probability of $\nu_{\tau}\left(\bar{\nu}_{\tau}\right)$ to appear as $\nu_{\mu}$ $\left(\bar{\nu}_{\mu}\right)$ at the detector is expressed by $P_{\tau \mu}\left(\bar{P}_{\tau \mu}\right)$. All the other symbols signify the same parameters as described in eq. (4.1). In our analysis, we take $\delta_{\mathrm{CP}}=0^{\circ}$ and therefore, we can write $P_{\alpha \beta}=P_{\beta \alpha}$ and $\bar{P}_{\alpha \beta}=\bar{P}_{\beta \alpha}$. Due to these properties and unitary nature of the PMNS matrix U [85-87], the sums of oscillation probabilities for neutrino and antineutrino in above equation become 1 . Therefore, $\nu_{\mu}$ and $\bar{\nu}_{\mu}$ event rates due to the dark matter annihilation/decay do not depend on the values of oscillation parameters.

In our simulation, the full three flavor neutrino oscillation probabilities are incorporated using the PREM profile for the Earth matter density [88]. The choices of central values of the oscillation parameters that are used in our simulation lie within the $1 \sigma$ range of these parameters as obtained from the recent global fit studies [89-91]. We produce all the results in this paper using the following benchmark values of oscillation parameters: $\sin ^{2} \theta_{23}=$ $0.5, \sin ^{2} 2 \theta_{13}=0.085, \Delta m_{\text {eff }}^{2}= \pm 2.4 \times 10^{-3} \mathrm{eV}^{2}, \sin ^{2} 2 \theta_{12}=0.84, \Delta m_{21}^{2}=7.5 \times 10^{-5} \mathrm{eV}^{2}$, and $\delta_{\mathrm{CP}}=0^{\circ}$. The $(+)$ and (-) signs of $\Delta m_{\mathrm{eff}}^{2}{ }^{8}$ correspond to normal ordering (NO) and

\footnotetext{
${ }^{7}$ The amount of $\nu_{e}, \bar{\nu}_{e}, \nu_{\mu}, \bar{\nu}_{\mu}, \nu_{\tau}$, and $\bar{\nu}_{\tau}$ fluxes from dark matter are same.

${ }^{8}$ The effective mass-squared difference, $\Delta m_{\text {eff }}^{2}$, is related to $\Delta m_{31}^{2}$ and $\Delta m_{21}^{2}$ through the expression $[92,93]$ :

$$
\Delta m_{\mathrm{eff}}^{2}=\Delta m_{31}^{2}-\Delta m_{21}^{2}\left(\cos ^{2} \theta_{12}-\cos \delta_{\mathrm{CP}} \sin \theta_{13} \sin 2 \theta_{12} \tan \theta_{23}\right) .
$$


inverted ordering (IO) respectively. In fit, we keep the values of oscillation parameters and the choice of mass ordering fixed.

In this analysis, we binned the $\nu$ and $\bar{\nu}$ data separately using reconstructed observables $E_{\nu}$ and $\cos \theta_{\nu}$ as described in table 3. There are total $29 E_{\nu}$ bins in the range of $E_{\nu}=[1$, $100] \mathrm{GeV}$. The bins of $E_{\nu}$ are chosen uneven to ensure that they are consistent with the energy resolution of the detector at various energy ranges. The isotropic nature of the signal allows us to take coarser binning in $\cos \theta_{\nu}$, and we take four $\cos \theta_{\nu}$ bins of equal size in the range $[-1,1]$. We use comparatively finer bins for reconstructed $\mathrm{E}_{\nu}$ because the signal has a strong dependency on energy of neutrino. We adopt an optimized binning scheme so that we have at least 2 events in each bin. The total number of bins used in our analysis is $29 \times 4=116$. We show the signal and background event distribution plots as a function of reconstructed neutrino energy for various $\cos \theta_{\nu}$ ranges in section 6 (see figures 4 and 6).

\section{Simulation method}

In our analysis, we consider the dark matter induced neutrinos as signal and treat atmospheric neutrinos as background. If $\mathrm{N}_{i j}^{a t m}$ and $\mathrm{N}_{i j}^{d m}$ denote the number of $\mu^{-}$events produced from the interactions of atmospheric $\nu_{\mu}$ and dark matter induced $\nu_{\mu}$ respectively in the $i$-th energy and $j$-th angular bin (see eqs. (4.1) and (4.4)), then the Poissonian $\chi^{2}$ [94] can be written as

$$
\begin{aligned}
\chi^{2}\left(\mu^{-}\right)= & \min _{\zeta_{\mathrm{atm}}, \zeta_{\mathrm{dm}}} \sum_{i=1}^{N_{E_{\nu}}} \sum_{j=1}^{N_{\cos \theta_{\nu}}} 2\left[N_{i j}^{\mathrm{th}}\left(\mu^{-}\right)-N_{i j}^{\exp }\left(\mu^{-}\right)-N_{i j}^{\exp }\left(\mu^{-}\right) \ln \frac{N_{i j}^{\mathrm{th}}\left(\mu^{-}\right)}{N_{i j}^{\exp }\left(\mu^{-}\right)}\right] \\
& +\zeta_{\mathrm{atm}}^{2}+\zeta_{\mathrm{dm}}^{2} .
\end{aligned}
$$

In the above equation, $N_{i j}^{\mathrm{exp}}=N_{i j}^{a t m}$ and $N_{i j}^{\mathrm{th}}=N_{i j}^{a t m}\left(1+\pi_{\mathrm{atm}} \zeta_{\mathrm{atm}}\right)+N_{i j}^{d m}\left(1+\pi_{\mathrm{dm}} \zeta_{\mathrm{dm}}\right)$ neglecting higher order terms. Here, $N_{E_{\nu}}=29$ and $N_{\cos \theta_{\nu}}=4$ as mentioned in table 3 . The quantities $\pi_{\mathrm{dm}}$ and $\pi_{\mathrm{atm}}$ in eq. (5.1) are the over all normalization errors on signal and background respectively. We take $\pi_{\mathrm{dm}}=\pi_{\mathrm{atm}}{ }^{9}=20 \%$. The systematic uncertainties in this analysis are incorporated using the pull method [96-98]. The parameters $\zeta_{\mathrm{dm}}$ and $\zeta_{\text {atm }}$ are the pull variables due to the systematic uncertainties on signal and background respectively. The values of $\zeta_{\mathrm{dm}}$ and $\zeta_{\mathrm{atm}}$ are obtained by setting $\frac{\partial \chi^{2}}{\partial \zeta_{\mathrm{dm}}}=0$ and $\frac{\partial \chi^{2}}{\partial \zeta_{\mathrm{atm}}}=0$, and their values lie within the range -1 to 1 . Following the same procedure, $\chi^{2}\left(\mu^{+}\right)$for $\mu^{+}$ events is obtained. We calculate the total $\chi^{2}$ by adding the individual contributions from $\mu^{-}$and $\mu^{+}$events in the following way ${ }^{10}$

$$
\chi_{\text {total }}^{2}=\chi^{2}\left(\mu^{-}\right)+\chi^{2}\left(\mu^{+}\right) .
$$

We notice that our results remain unchanged if we consider larger uncertainties on the atmospheric neutrino events. The reason behind this is that for any choice of $m_{\chi}$ we have many bins in terms of the reconstructed observables $\mathrm{E}_{\nu}$ and $\cos \theta_{\nu}$, which are not affected

\footnotetext{
${ }^{9}$ For a detailed discussion on the uncertainties of the atmospheric neutrino flux, see ref. [95].

${ }^{10}$ Here, we would like to mention that though we assume same amount of normalization uncertainties for $\mu^{-}$and $\mu^{+}$events, we get different values of $\zeta_{\mathrm{dm}}$ and $\zeta_{\mathrm{atm}}$ for $\mu^{-}$and $\mu^{+}$channels.
} 
by the dark matter induced neutrinos. Therefore these bins can constrain the uncertainties on the atmospheric neutrino flux. On the other hand, we notice that if we take the larger uncertainties on the dark matter induced neutrino events, say $30 \%$, our final results get modified by 2 to $3 \%$. It is worthwhile to mention that the maximum uncertainty on the signal stems from the dark matter density profile. Therefore, we give our results assuming two different profiles for the dark matter density which are the NFW and the Burkert.

As we have discussed in section 4 , the dark matter induced signal does not depend on the oscillation parameters as long as we take the CP-violating phase $\delta_{\mathrm{CP}}=0^{\circ}$. The dependency on the oscillation parameters in the results comes only through the atmospheric neutrino background. We produce all the results assuming normal ordering both in data and theory. We have checked that the results hardly change if we consider inverted ordering. One of the main reasons behind this is that due to our choice of coarser reconstructed $\cos \theta_{\nu}$ bins, the information coming from the MSW effect [99-102] in the atmospheric neutrino events gets smeared out substantially. Another reason is that since the dark matter induced neutrino signal appears only in 2 to $3 E_{\nu}$ bins (see in figures 4 and 6), $\chi^{2}$ is hardly affected due to the change in atmospheric neutrino background in these bins when we switch from NO to IO.

\section{Results}

\subsection{Constraints on annihilation of dark matter}

In this section, we present the constraints on self-annihilation cross-section of dark matter $(\chi \chi \rightarrow \nu \bar{\nu})$ which can be obtained by $500 \mathrm{kt} \cdot \mathrm{yr}$ of MagICAL exposure. The background consists of conventional atmospheric neutrinos, and the signal consists of neutrinos coming from dark matter annihilation. The simulated event spectra as a function of reconstructed neutrino energy in $500 \mathrm{kt} \cdot \mathrm{yr}$ exposure of MagICAL detector are presented in figure 4 . The quantity in the y-axis of figure 4 is the number of events per unit energy range multiplied by the mid value in each energy bin. In each panel, the black solid line represents the event distribution of conventional atmospheric $\nu_{\mu}$, denoted by ATM. If DM particles of mass $30 \mathrm{GeV}$, for example, self-annihilate to $\nu \bar{\nu}$ pairs, then each of these $\nu$ and $\bar{\nu}$ will have $30 \mathrm{GeV}$ of energy. The total neutrino event spectra in MagICAL detector in presence of DM annihilation are shown by the red dotted lines (ATM + DM) in figure 4 . The value of selfannihilation cross-section of dark matter for these plots is taken to be $3.5 \times 10^{-23} \mathrm{~cm}^{3} \mathrm{~s}^{-1}$.

An excess of $\nu_{\mu}$ events due to dark matter annihilation appears over the ATM around reconstructed neutrino energy of $30 \mathrm{GeV}$. These events get distributed over nearby energy bins due to the finite energy resolution of the detector. The number of signal and atmospheric events in neutrino mode are 174 and 210 respectively in the energy range [25, 35] $\mathrm{GeV}$ and $\cos \theta_{\nu} \in[-1,1]$. There are 4 panels: each represents the event distribution summed over different $\cos \theta_{\nu}$ ranges. The figures in top panels portray the event spectra over $\cos \theta_{\nu} \in[-1,-0.5]$ and $[-0.5,0.0]$ from left to right. These events are due to upward going neutrinos, which travel a long distance through the Earth matter before they reach the detector. Though in these panels, the signatures of neutrino flavor oscillation are seen in ATM spectra, but the imprints of the Earth matter effect are not visible due to the choice 

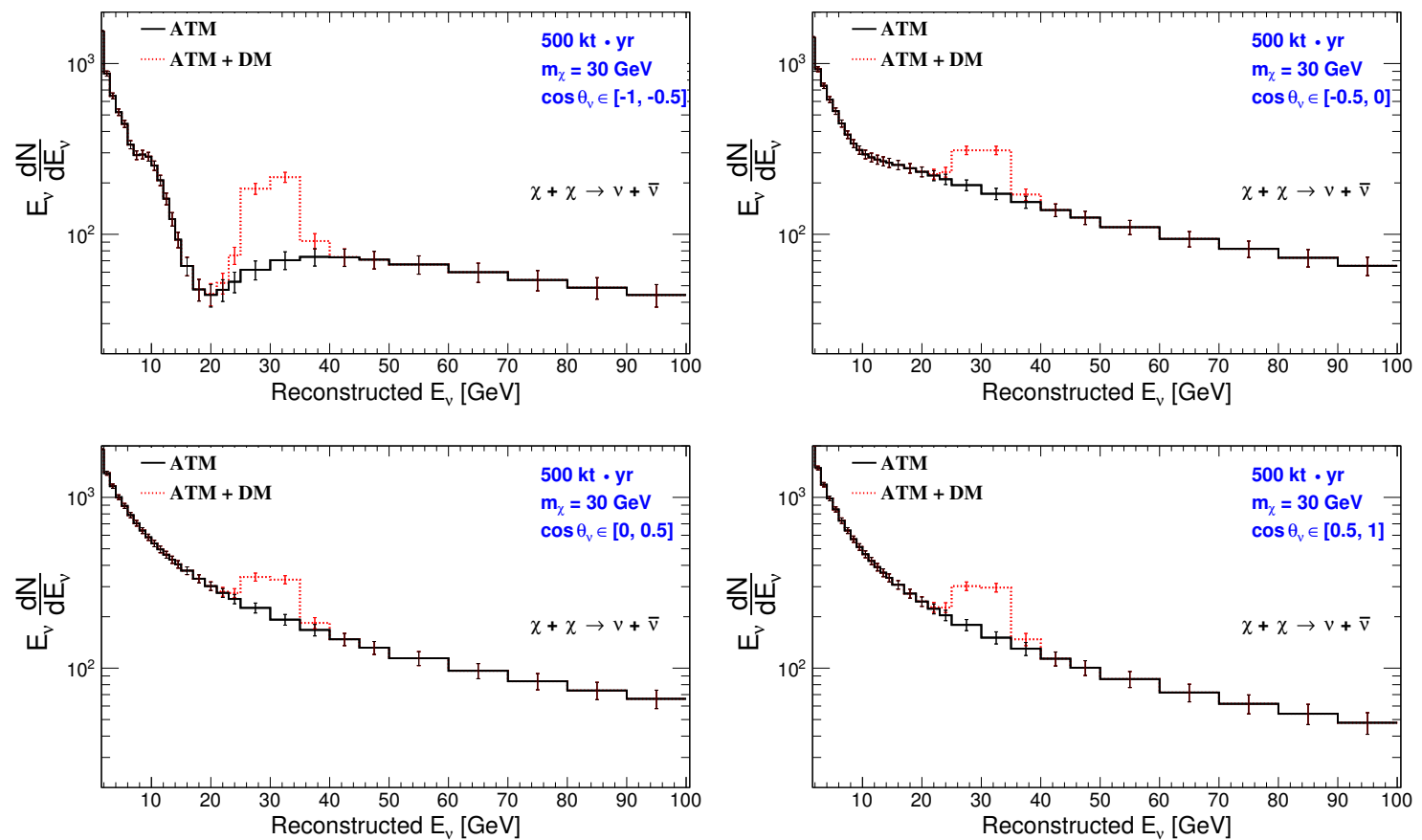

Figure 4. Event spectra of atmospheric $\nu_{\mu}$ (denoted by ATM) are shown by the black solid lines. The predicted event distributions coming from atmospheric $\nu_{\mu}$ and dark matter originated neutrino $(\mathrm{ATM}+\mathrm{DM})$ are shown in red dotted lines for different $\cos \theta$ ranges using $500 \mathrm{kt} \cdot \mathrm{yr}$ exposure of the MagICAL detector. The signal (DM) is coming from $30 \mathrm{GeV}$ annihilating DM particles here. The mass ordering is taken as NO. The $\chi \chi \rightarrow \nu \bar{\nu}$ cross-section is arbitrarily chosen to $\langle\sigma v\rangle=3.5 \times 10^{-23} \mathrm{~cm}^{3} \mathrm{~s}^{-1}$ to have visual clarity.

of our large $\cos \theta_{\nu}$ bins. The energy distributions of downward going events are shown in bottom panels from left to right for $\cos \theta_{\nu} \in[0.0,0.5]$ and $[0.5,1.0]$ respectively. These neutrinos do not oscillate as they traverse a length smaller than the oscillation wavelength in multi-GeV range. The statistical error bars in these plots associated with different energy bins are equal to the square root of the number of events in the corresponding bins.

The charge identification ability ${ }^{11}$ of the MagICAL detector provides an opportunity to explore the same physics in neutrino and antineutrino channels separately. This is not possible in water Cherenkov, liquid scintillator, and liquid argon based detectors. The MagICAL detector will have separate data sets for $\nu_{\mu}$ and $\bar{\nu}_{\mu}$. The total sensitivity is obtained by combining the $\nu_{\mu}$ and $\bar{\nu}_{\mu}$ data sets according to eq. (5.2). We present results by using $\nu_{\mu}$ and $\bar{\nu}_{\mu}$ data separately, and then combining these two. The upper limits on self-annihilation cross-section $(\langle\sigma v\rangle)$ of DM particles for the process $\chi \chi \rightarrow \nu \bar{\nu}$ at 90\% C.L.

\footnotetext{
${ }^{11}$ We have checked that $\chi_{\nu}^{2}+\chi_{\bar{\nu}}^{2}$ is better than $\chi_{\nu+\bar{\nu}}^{2}$ by very little amount, which is around $2 \%$. In our analysis, CID does not play an important role unlike in the case of mass ordering determination for the following reasons. First, the signal is independent of oscillation parameters and it appears only in two to three $E_{\nu}$ bins. Secondly, the impact of the Earth matter effect in atmospheric $\nu$ and $\bar{\nu}$ events (background) gets reduced for our choices of large $\cos \theta_{\nu}$ bins.
} 


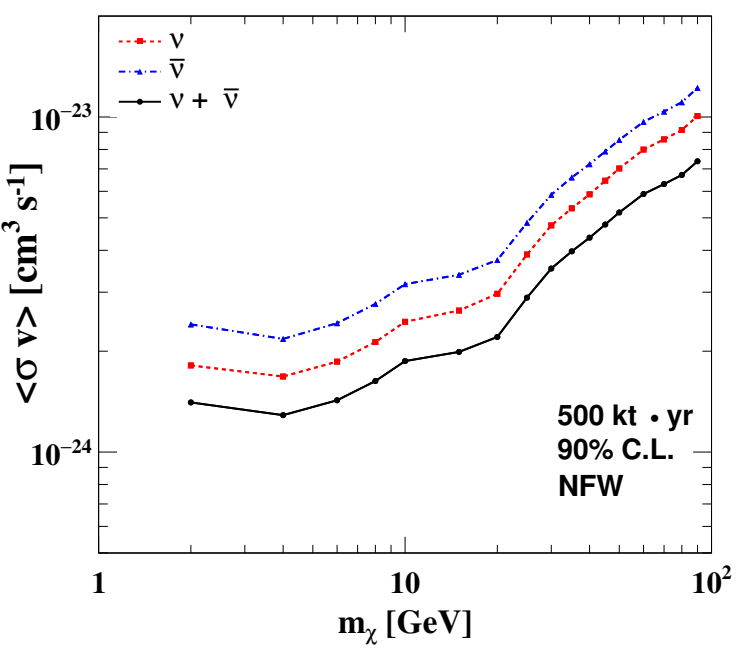

(a)

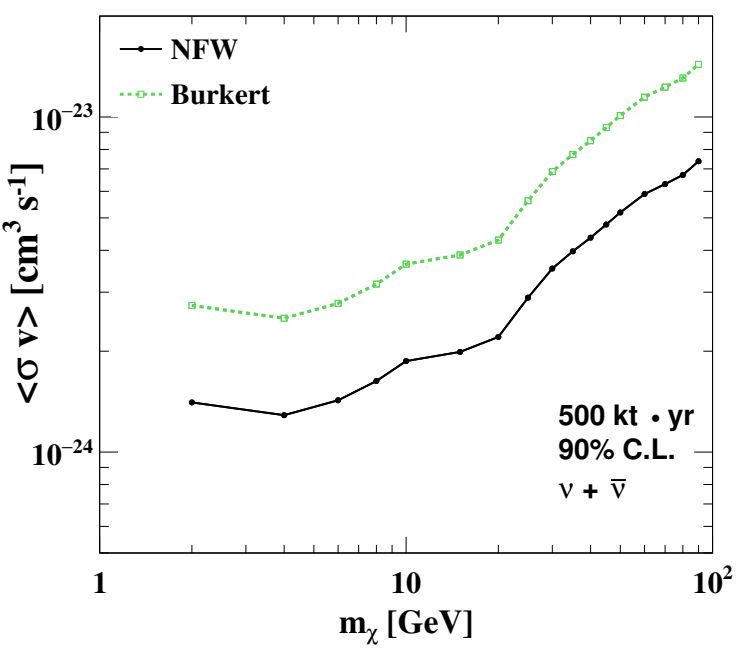

(b)

Figure 5. (a) The upper limit on self-annihilation cross-section of DM particle $(\chi \chi \rightarrow \nu \bar{\nu})$ at $90 \%$ C.L. (1 d.o.f.) as a function of DM mass $m_{\chi}$ using $500 \mathrm{kt} \cdot \mathrm{yr}$ exposure of the MagICAL detector. The bound calculated with only $\nu_{\mu}\left(\bar{\nu}_{\mu}\right)$-induced events is shown with red dashed (blue dot-dashed) line as MagICAL can distinguish $\nu_{\mu}$ from $\bar{\nu}_{\mu}$. The upper bound obtained by combining these two channels is also shown by the black solid line in the figure. We take the NFW as DM profile. (b) The upper bounds on the self-annihilation cross-section $(\chi \chi \rightarrow \nu \bar{\nu})$ of dark matter are presented for the NFW (black solid) and Burkert (green dashed) profiles combining the information coming from $\nu_{\mu}$ and $\bar{\nu}_{\mu}$. For both (a) and (b), the choice of mass ordering is NO.

(1 d.o.f.) that MagICAL will obtain with 10 years of data are represented in figure 5 . The red dashed, blue dot-dashed, and the black solid lines in figure 5(a) represent the limits on $\langle\sigma v\rangle$ from $\nu_{\mu}, \bar{\nu}_{\mu}$, and the combination of $\nu_{\mu}$ and $\bar{\nu}_{\mu}$ data respectively using the NFW profile. Analysis with $\nu_{\mu}$ gives tighter bound than $\bar{\nu}_{\mu}$ because of the higher statistics of $\nu_{\mu}$ over $\bar{\nu}_{\mu}$.

At higher energies, the atmospheric neutrino flux (background) decreases, and same happens to the signal coming from dark matter self annihilation because of its $m_{\chi}^{-2}$ dependence (see eq. (2.6)). A competition between these two effects lowers the signal to background ratio for heavy dark matter particles. Thus, the bound on $\langle\sigma v\rangle$ becomes weaker for heavy DM. We can have a rough estimate of how $\langle\sigma v\rangle$ depends on $m_{\chi}$ in the range say 4 to $8 \mathrm{GeV}$ by mainly considering the energy dependence of flux and interaction cross-section in both signal and background. In this $m_{\chi}$ range which also corresponds to neutrino energy range of 4 to $8 \mathrm{GeV}$, the atmospheric flux varies as $\sim E_{\nu}^{-2.7}$, whereas neutrinos flux from the annihilating DM goes as $\langle\sigma v\rangle / m_{\chi}^{2}$. For both signal and background, the neutrino-nucleon CC cross-section is approximately proportional to $E_{\nu}$ or $m_{\chi}$ in case of annihilation. Therefore, the neutrino signal from dark matter annihilation $(S)$ depends on $m_{\chi}$ in the following way: $S \propto \frac{\langle\sigma v\rangle}{m_{\chi}^{2}} \cdot m_{\chi}=\langle\sigma v\rangle / m_{\chi}$. As far as background $(B)$ is concerned, $B \propto m_{\chi}^{-2.7} \cdot m_{\chi}=m_{\chi}^{-1.7}$. Hence, in case of annihilation, $S / \sqrt{B} \propto\langle\sigma v\rangle \cdot m_{\chi}^{-0.15}$ or, $\langle\sigma v\rangle \propto m_{\chi}^{0.15}$ if $S / \sqrt{B}$ remains constant. From figure $5\left(\right.$ a), we can see that at $m_{\chi}=$ $4 \mathrm{GeV}$, the limit on $\langle\sigma v\rangle$ is $1.2 \times 10^{-24} \mathrm{~cm}^{3} \mathrm{~s}^{-1}$ in case of $\nu+\bar{\nu}$ modes. Now, from our 
approximate expression as mentioned above, the limit on $\langle\sigma v\rangle$ at $m_{\chi}=8 \mathrm{GeV}$ should be around $1.2 \times 10^{-24} \times(8 / 4)^{0.15} \mathrm{~cm}^{3} \mathrm{~s}^{-1}=1.33 \times 10^{-24} \mathrm{~cm}^{3} \mathrm{~s}^{-1}$, which is indeed the case as can be seen from the black solid line in figure $5(\mathrm{a})$. If we want to do the same exercise for $m_{\chi}<4 \mathrm{GeV}$, then the only change that we have to make is that the atmospheric neutrino flux varies as $E_{\nu}^{-2}$ at those energies instead of $E_{\nu}^{-2.7}$. On the other hand, to explain the nature of the same curve for $m_{\chi}$ above $8 \mathrm{GeV}$, we have to also take into account the effect of neutrino flavor oscillation and detector response which have nontrivial dependence on $E_{\nu}$ whereas, the atmospheric neutrino flux still varies as $E_{\nu}^{-2.7}$ in this energy range.

We compare the constraints with the NFW and the Burkert profiles by black solid and green dashed lines respectively in figure 5(b) combining the neutrino and antineutrino data. We obtain better sensitivity with the NFW profile than with the Burkert profile. The average value of $\mathcal{J}$ factor over $4 \pi$ solid angle for the Burkert profile is smaller than that for the NFW profile. Thus, the signal strength with Burkert profile is smaller than that with the NFW profile. We have $\mathcal{J}_{\Delta \Omega}^{\text {ann }}=3.33$ and 1.60 for the NFW and Burkert profiles respectively, with $\Delta \Omega=4 \pi$.

\subsection{Constraints on decay of dark matter}

Assuming that dark matter particles have a mass of $30 \mathrm{GeV}$, and they decay to $\nu \bar{\nu}$ pairs, then the energy that each $\nu$ and $\bar{\nu}$ carries is $15 \mathrm{GeV}$. These events give rise to an excess of $\nu_{\mu}$ and $\bar{\nu}_{\mu}$ events around reconstructed neutrino energy of $15 \mathrm{GeV}$ on top of the atmospheric neutrino event distribution as shown in figure 6 . The black solid lines represent the event distributions for the atmospheric neutrinos and the red dotted lines show event distributions for background along with the signal. The four panels in figure 6 correspond to different $\cos \theta_{\nu}$ ranges as mentioned in the figure legends. Here, we assume the lifetime $(\tau)$ of dark matter to be $4.7 \times 10^{24} \mathrm{~s}$ and we take $500 \mathrm{kt} \cdot \mathrm{yr}$ exposure for the MagICAL detector. We can see from figure 6 that the events due to the decay of dark matter get distributed around $15 \mathrm{GeV}$ due to the finite energy resolution of detector. In this case, the number of the signal and background events are 81 and 289 respectively in the reconstructed energy range [13, 17] $\mathrm{GeV}$ and $\cos \theta_{\nu} \in[-1,1]$.

The future sensitivity of the MagICAL detector to set a lower limit on the lifetime $(\tau)$ of dark matter as a function of $m_{\chi}$ is shown in figure 7 . We give the results at $90 \%$ C.L. (1 d.o.f.) assuming $500 \mathrm{kt} \cdot \mathrm{yr}$ exposure of the proposed MagICAL detector. Here, we assume the dark matter density profile to be the NFW. The red dashed (blue dotdashed) line in figure 7 (a) represents the bound which we obtain using $\nu_{\mu}\left(\bar{\nu}_{\mu}\right)$ data set. The bound gets improved when we add the $\nu_{\mu}$ and $\bar{\nu}_{\mu}$ data sets and the corresponding result is shown by the black solid line. Here, we see that the limits on the dark matter lifetime get improved when we consider higher $\mathrm{m}_{\chi}$. It happens for the following reasons. The flux of neutrinos coming from the dark matter decay (signal) has a $m_{\chi}^{-1}$ dependence (see eq. (2.11)) and the atmospheric neutrino flux (background) gets reduced substantially at higher energies. We find that in presence of these two competing effects, ultimately, the signal over background ratio gets improved for higher $m_{\chi}$, which allows us to place restrictive bounds on the lifetime of dark matter. In figure 7(a), we can explain how the limit on dark matter life time $\tau$ depends on $m_{\chi}$ in the range say $8 \mathrm{GeV} \leq m \chi \leq 16 \mathrm{GeV}$ 

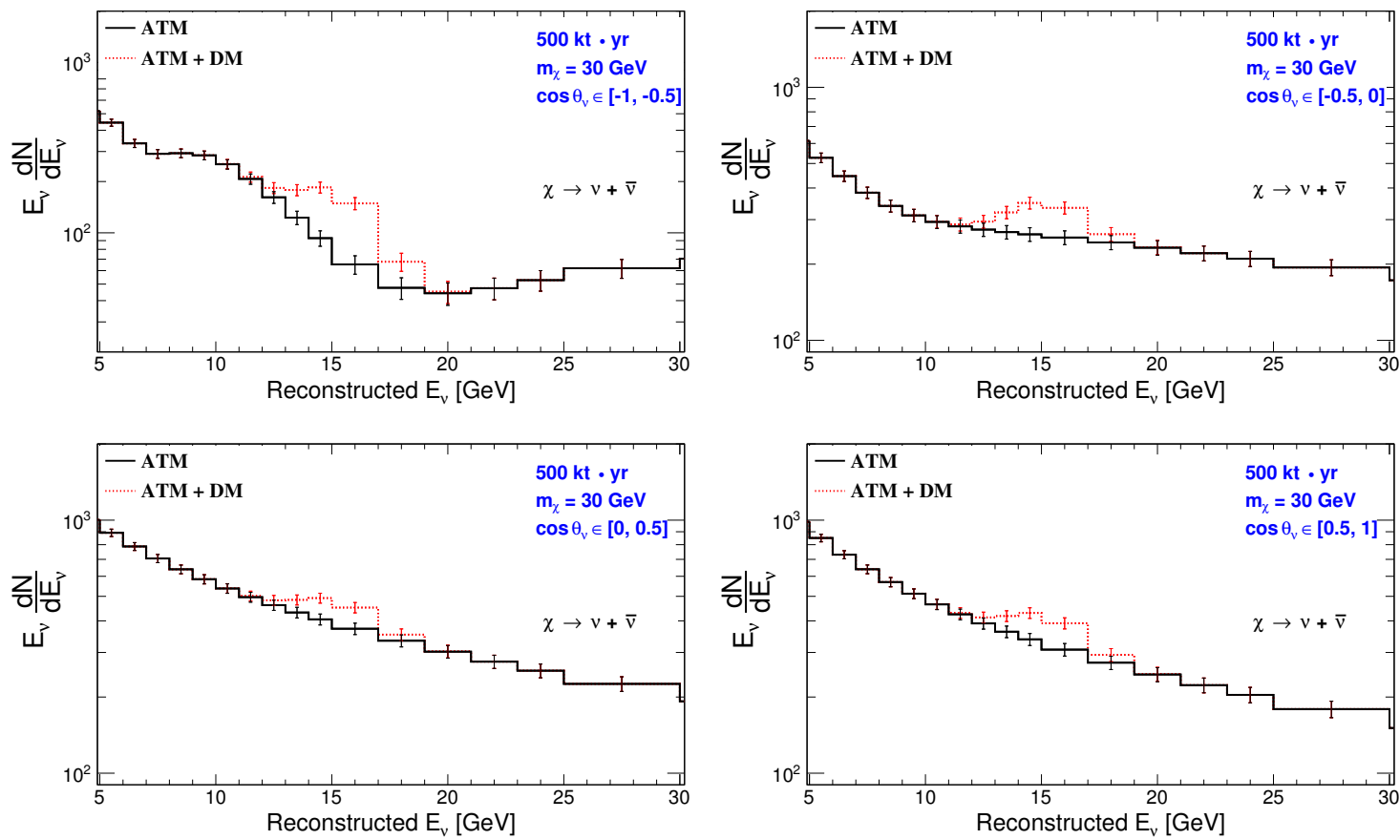

Figure 6. The event distribution of atmospheric $\nu_{\mu}$ (denoted as ATM) and the predicted $\nu_{\mu}$ event spectra in presence of decay of $30 \mathrm{GeV}$ dark matter particles (denoted as ATM + DM) in different $\cos \theta_{\nu}$ ranges using $500 \mathrm{kt} \cdot \mathrm{yr}$ exposure of the MagICAL detector. Black solid (red dotted) line represents the ATM (ATM + DM). The mass ordering is taken as NO. The lifetime of dark matter is arbitrarily chosen $\left(4.7 \times 10^{24} \mathrm{~s}\right)$ for sake of visual clarity.

by mainly taking into account the energy dependence of the flux and neutrino-nucleon $\mathrm{CC}$ cross-section in the same fashion which adopt to explain the bound on $\langle\sigma v\rangle$ in the previous section. The above range of $m_{\chi}$ corresponds to the $E_{\nu}$ range of $4 \mathrm{GeV}$ to $8 \mathrm{GeV}$, since the neutrino energy from decaying $\mathrm{DM}$ is $E_{\nu}=m_{\chi} / 2$. Here, the neutrino flux from decaying DM is proportional to $\frac{1}{m_{\chi} \tau}$ (see eq. (2.11)). Thus, the neutrino signal $(S)$ from dark matter decay varies as $S \propto \frac{1}{m_{\chi} \tau} \cdot m_{\chi}=1 / \tau$, while the background varies with $m_{\chi}$ in the same way as we see in case of annihilation which is $B \propto m_{\chi}^{-1.7}$. Hence, in case of decaying DM, $S / \sqrt{B} \propto \frac{1}{\tau} \cdot m_{\chi}^{0.85}$ or, $\tau \propto m_{\chi}^{0.85}$ for a fixed value of $S / \sqrt{B}$. From figure 7 (a), it can be seen that at $m_{\chi}=8 \mathrm{GeV}$, the limit on $\tau$ is $4.0 \times 10^{24} \mathrm{~s}$ combining $\nu$ and $\bar{\nu}$ modes. From the simple $m_{\chi}$ dependence of $\tau$ that we discuss above, at $16 \mathrm{GeV}$, the limit on $\tau$ should be around $4.0 \times 10^{24} \times(16 / 8)^{0.85} \mathrm{~s}=7.21 \times 10^{24} \mathrm{~s}$, which is very close to the value as can be seen from the black solid line in figure $7(\mathrm{a})$. To obtain the similar analytical understanding for $m_{\chi}$ below $8 \mathrm{GeV}$, we need to make suitable changes in the energy dependence of atmospheric neutrino flux which we have already discussed in the previous section. Similarly, to see how $\tau$ varies with $m_{\chi}$ above $16 \mathrm{GeV}$, we have to also take into account the nontrivial energy dependence of neutrino flavor conversion and detector response along with flux and cross-section. 


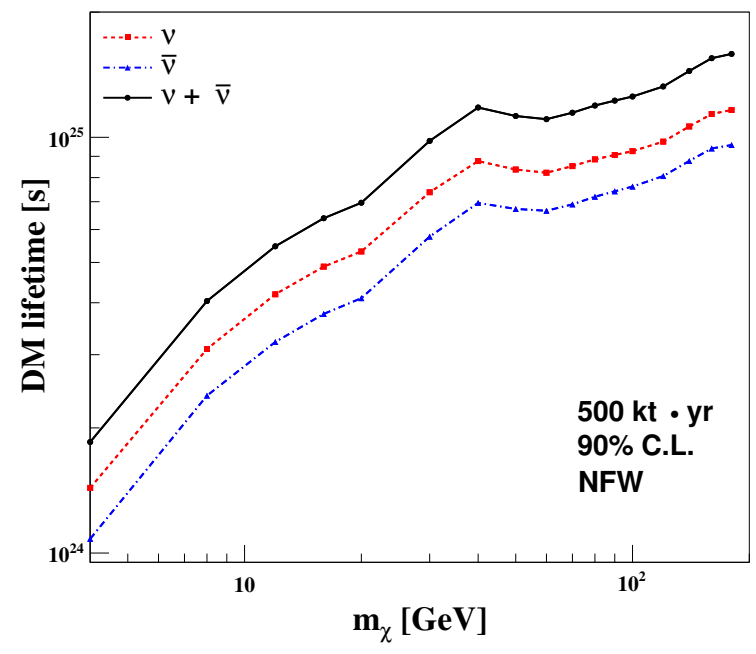

(a)

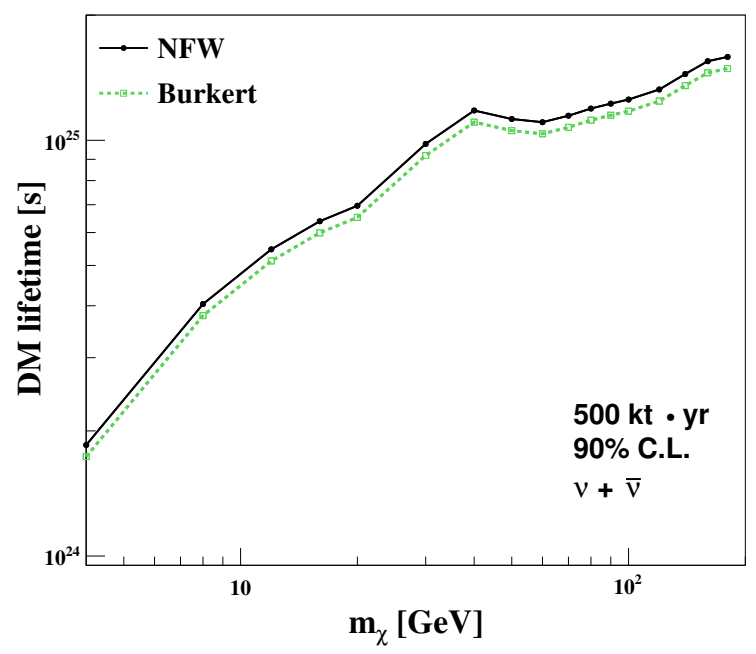

(b)

Figure 7. (a) The lower bound on the decay lifetime of dark matter $(\chi \rightarrow \nu \bar{\nu})$ as a function of DM mass $m_{\chi}$ at $90 \%$ C.L. (1 d.o.f.) obtained using only $\nu_{\mu}$ and only $\bar{\nu}_{\mu}$ data using $500 \mathrm{kt} \cdot \mathrm{yr}$ exposure of MagICAL. The red dashed (blue dot-dashed) line shows the sensitivity coming from $\nu_{\mu}\left(\bar{\nu}_{\mu}\right)$-induced events. The black solid line represents the same combining $\nu_{\mu}$ and $\bar{\nu}_{\mu}$ events at $\chi^{2}$ level. We take NO as mass ordering. (b) The constraints on the decay lifetime of dark matter $(\chi \rightarrow \nu \bar{\nu})$ assuming the NFW (black solid line) and Burkert (green dashed line) profiles using 500 $\mathrm{kt} \cdot \mathrm{yr}$ of MagICAL exposure. Here the results are shown combining $\nu$ and $\bar{\nu}$ (see section 5 for detail).

Due to the uncertainties in the dark matter density profiles, we present the bound on decay lifetime of dark matter with the profiles: NFW and Burkert by the black solid and green dashed line respectively in figure 7(b). Ref. [103] considers only $\mu^{+} \mu^{-}$as final states for dark matter decay in the context of ICAL-INO, although their constraints are much weaker.

\subsection{Comparison with other experiments}

Various experiments present the bounds on the self-annihilation cross-section of $\chi \chi \rightarrow \nu \bar{\nu}$ and the decay lifetime of $\chi \rightarrow \nu \bar{\nu}$ processes. Figure 8(a) shows a comparison of the current bounds on $\langle\sigma v\rangle$ at $90 \%$ C.L. (1 d.o.f.) from the first three phases of the SuperKamiokande [21] (blue long-dashed line), the four phases of the Super-Kamiokande [104] (blue long-dash-dotted line), IceCube [65, 105] (green dot-dashed and green triple-dotdashed lines), ANTARES [106, 107] (red dotted and red dashed lines), PINGU [108] (green shade), and from the MagICAL detector (black solid line) for the process $\chi \chi \rightarrow \nu \bar{\nu}$. We do not show the weaker limits from Baikal NT200 [109]. In figure 8(b), we compare the limits on decay lifetime $(\tau)$ for the process $\chi \rightarrow \nu \bar{\nu}$ from the first three phases of the SuperKamiokande experiment [21] (blue long-dashed line) and the present work (black solid line).

Due to the lower energy threshold of MagICAL, the dark matter constraints can be estimated for $m_{\chi}$ values which are as low as $2 \mathrm{GeV}$ and $4 \mathrm{GeV}$ in case of annihilating and decaying dark matter respectively. The good energy and direction resolutions of MagI- 


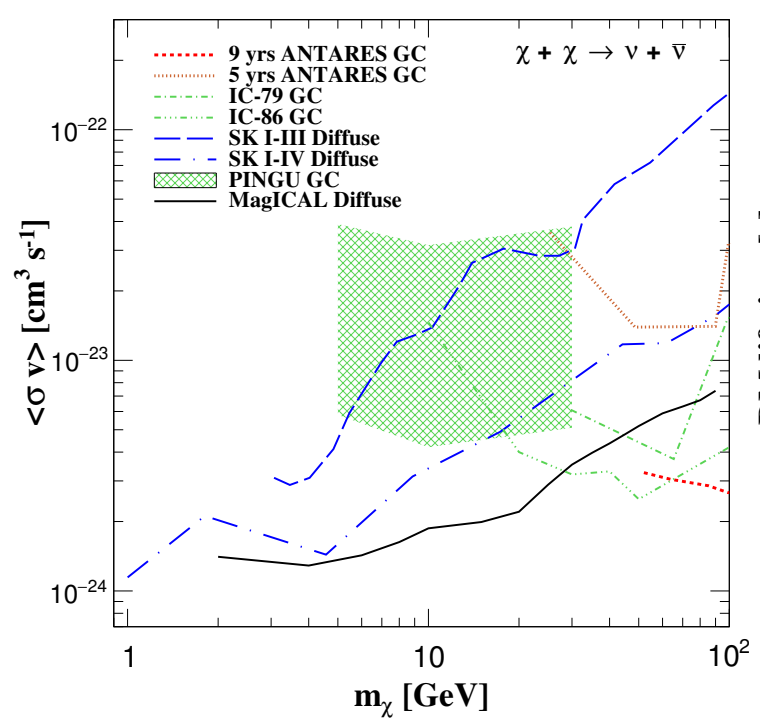

(a)

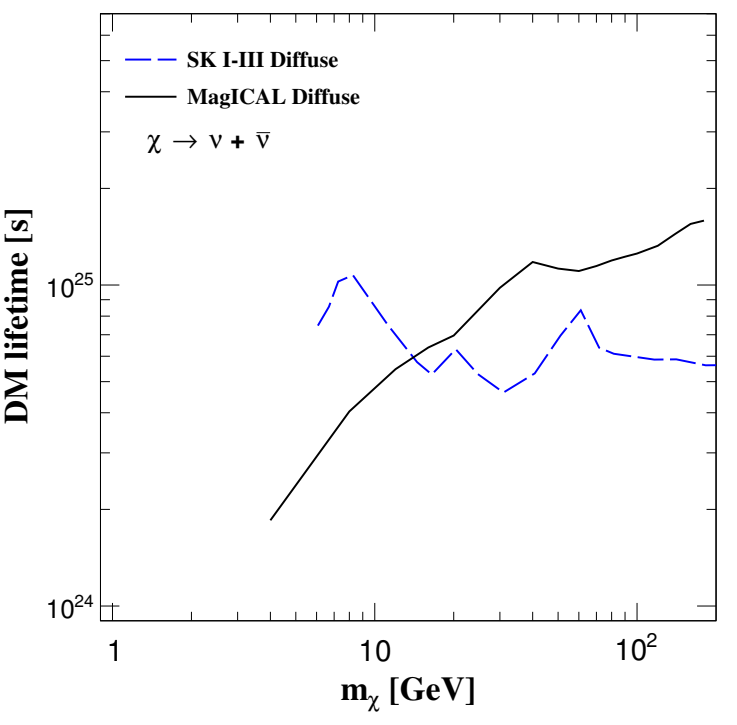

(b)

Figure 8. (a) Current bounds at $90 \%$ C.L. (1 d.o.f.) on self-annihilation cross-section which are obtained from the first three phases of Super-Kamiokande [21] (blue long-dashed line), the four phases of Super-Kamiokande [104] (blue long-dash-dotted line), IceCube [65, 105] (green dot-dashed and green triple-dot-dashed lines), and ANTARES [106, 107] (red dotted and red dashed lines) are shown. The future sensitivity of PINGU [108] with its 1 year of exposure is shown by green shaded region. We compare these limits with the bound obtained from $500 \mathrm{kt} \cdot \mathrm{yr}$ MagICAL (black solid line) detector. For all the cases the NFW profile is used. (b) Blue long-dashed line shows the current bound on decay lifetime of DM from the first three phases of Super-Kamiokande [21] using the NFW profile. We compare this limit with the performance of $500 \mathrm{kt} \cdot \mathrm{yr}$ MagICAL detector (black solid line) using the same NFW profile.

CAL detector help to strongly constrain the $\langle\sigma v\rangle$ and $\tau$ for $m_{\chi}$ in multi-GeV range. The constraints on $\langle\sigma v\rangle$ obtained using 319.7 live-days of data from IceCube operating in its 79 string configuration during 2010 and 2011 are stronger than MagICAL for dark matter masses heavier than $\sim 50 \mathrm{GeV}$ (see green dot-dashed line in figure 8(a)) [24, 65, 110119]. But, if we consider the limits on $\langle\sigma v\rangle$ estimated using three years of the IceCube/DeepCore data [105], then their performance becomes better than the MagICAL detector for $m_{\chi} \geq 30 \mathrm{GeV}$ (see green triple-dot-dashed line in figure $8(\mathrm{a})$ ). Using the 9 years data of ANTARES, no excess was found over the expected neutrino events in the range of WIMP mass $50 \mathrm{GeV} \leq m_{\chi} \leq 100 \mathrm{GeV}$, and they presented the most stringent constraint on $\langle\sigma v\rangle$ for $m_{\chi} \geq 70 \mathrm{GeV}$ [107]. However, for dark matter masses $\lesssim 100 \mathrm{GeV}$, the potential constraints from MagICAL are comparable or slightly better than that from Super-Kamiokande [21, 104]. The limit on $\langle\sigma v\rangle$ by $500 \mathrm{kt} \cdot \mathrm{yr}$ exposure of MagICAL detector is better than that from 1 year exposure of PINGU [108]. The constraints on dark matter annihilation and decay that we show in figure 8 can only be obtained from neutrino telescopes, including liquid scintillator detectors [120, 121]. The dark matter masses that we consider are too low for efficient electroweak bremsstrahlung, and hence gamma-ray 


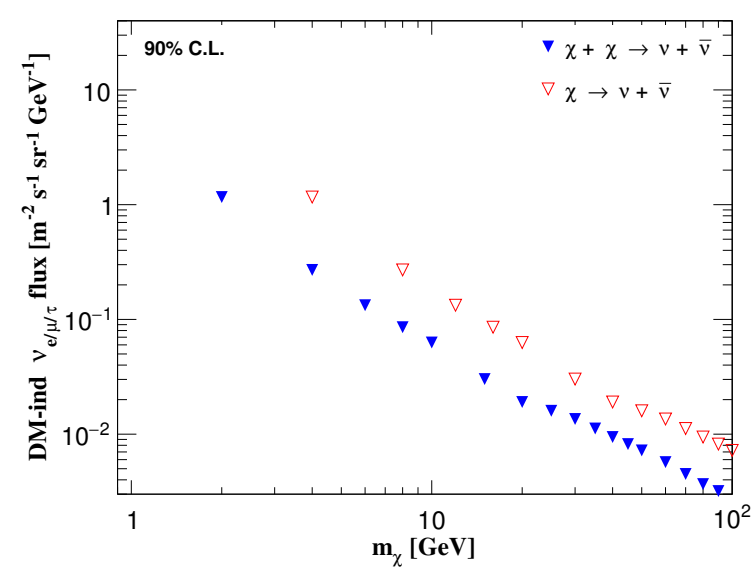

(a)

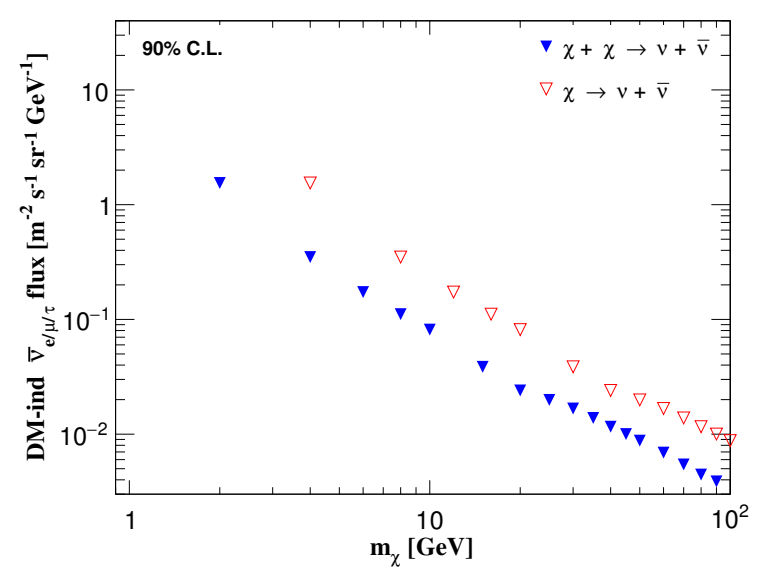

(b)

Figure 9. The limit on (a) $\nu_{e / \mu / \tau}$ and (b) $\bar{\nu}_{e / \mu / \tau}$ flux produced from the dark matter in Milky Way galaxy at $90 \%$ C.L. (1 d.o.f.) by $500 \mathrm{kt} \cdot \mathrm{yr}$ MagICAL detector. The blue filled and red empty triangles are for the annihilation and decay of dark matter particles respectively.

constraints on this channel are weak [122-130]. Since MagICAL can distinguish between $\mu^{+}$and $\mu^{-}$, it can also give constraints on exotic lepton number violating dark matter interactions. The potential dark matter constraints from Baikal-GVD, and Hyper-Kamiokande will be stronger or comparable [131, 132]. The complementarity of INO-MagICAL with PINGU and Hyper-Kamiokande will certainly make dark matter physics richer.

\subsection{The constraints on DM-induced neutrino flux}

We can use the constraints on $\langle\sigma v\rangle$ (see section 6.1) and $\tau$ (see section 6.2) in eqs. (2.6) and (2.11) respectively to place the upper bound on the neutrino and antineutrino flux from dark matter . In figure 9(a), the blue filled triangles and red empty triangles depict the upper bounds on $\nu_{e} / \nu_{\mu} / \nu_{\tau}$ flux at $90 \%$ C.L. (1 d.o.f.) using the constraints on $\langle\sigma v\rangle$ (in case of annihilation) and $\tau$ (in case of decay) respectively. Figure 9 (b) shows the same for $\bar{\nu}_{e} / \bar{\nu}_{\mu} / \bar{\nu}_{\tau}$ flux. The mass ordering is taken as NO and the dark matter profile is assumed to be NFW. We can see from both the panels in figure 9 that the limits on neutrino (left panel) and antineutrino (right panel) flux from both annihilation and decay improve as we increase the value of $m_{\chi}$. We can understand this behavior in the following way. We know that the atmospheric neutrino event rates which serve as background for annihilation and decay decrease as we go to higher neutrino energy. This can be clearly seen from figure 4 and also figure 6 . This is also true for atmospheric antineutrino events. Since, the atmospheric neutrino and antineutrino backgrounds get reduced when we go from lower to higher $m_{\chi}$, we need less dark matter induced neutrino and antineutrino flux for both annihilation and decay to obtain the same confidence level in $\Delta \chi^{2}$ which is 2.71 at $90 \%$ C.L. (1 d.o.f). Hence, we can place better constraints on the DM induced neutrino and anitneutrino flux as we move from lower to higher $m_{\chi}$ values. Another feature that is emerging from both the panels in figure 9 that we have better constraints on the neutrino and antineutrino flux obtained from the annihilation of dark matter as compare to its decay for a fixed $m_{\chi}$. We can also explain this 
feature in the following way. For a fixed value of $m_{\chi}$, the available energy of neutrino and antineutrino, $E_{\nu / \bar{\nu}}$, is equal to $m_{\chi}$ for annihilation and $m_{\chi} / 2$ for decay. Let us consider the case for $m_{\chi}=10 \mathrm{GeV}$ in both the panels. In this case, the available neutrino/antineutrino energy for annihilation (decay) is $10 \mathrm{GeV}(5 \mathrm{GeV})$. Now, we already know that the background events induced by atmospheric neutrino and anitneutrino flux are higher at $5 \mathrm{GeV}$ (in case of decay) as compared to $10 \mathrm{GeV}$ (in case of annihilation). Therefore, for a fixed choice of $m_{\chi}$ value, we need higher neutrino and antineutrino flux from decaying DM as compare to annihilating DM to place the constraints at same confidence level.

\section{Conclusions}

We explore the prospects of detecting diffuse dark matter in the Milky Way galaxy at the proposed INO-MagICAL detector. The future sensitivity of $500 \mathrm{kt} \cdot \mathrm{yr}$ MagICAL detector to constrain the dark matter self-annihilation cross-section $(\langle\sigma v\rangle)$ and decay lifetime $(\tau)$ for $\chi \chi \rightarrow \nu \bar{\nu}$ and $\chi \rightarrow \nu \bar{\nu}$ processes respectively are estimated. We find that MagICAL will be able to probe new parameter space for low mass dark matter.

Combining information from $\nu$ and $\bar{\nu}$ modes, the future limits on $\langle\sigma v\rangle$ and $\tau$ are $\leq 1.87$ $\times 10^{-24} \mathrm{~cm}^{3} \mathrm{~s}^{-1}$ and $\geq 4.8 \times 10^{24} \mathrm{~s}$ respectively at $90 \%$ C.L. (1 d.o.f.) for $m_{\chi}=10 \mathrm{GeV}$ assuming the NFW profile. These limits will be novel and they will address many viable dark matter models. The limits for higher dark matter masses will also be competitive with other neutrino telescopes.

We have also shown the bounds on $\langle\sigma v\rangle$ and $\tau$ with $\nu$ and $\bar{\nu}$ data separately. This enables us to probe the same physics through the $\nu$ and $\bar{\nu}$ channels due to the charge identification capability of the MagICAL detector.

Although, we have studied the processes $\chi \chi \rightarrow \nu \bar{\nu}$ and $\chi \rightarrow \nu \bar{\nu}$, other final states like $\mu^{+} \mu^{-}, \tau^{+} \tau^{-}, b \bar{b}$ are also possible. The constraints on these channels obtained from the gamma-ray detectors are much stronger, and hence we do not consider them. Since the analysis is done for the diffuse dark matter component of the Milky Way galaxy, the constraints on self-annihilation cross-section and decay lifetime are robust and conservative, and the constraints have mild dependence on the dark matter profile. Besides new and novel methods in dark matter indirect detection physics $[133,134]$, it is imperative that we fully utilize the capabilities of new and upcoming detectors. Our work explores the capabilities of INO-MagICAL to search for dark matter, and we encourage the community to look into this signature in more detail.

\section{Acknowledgments}

A.K. would like to thank the INO project for financial support. R.L. thanks KIPAC for financial help. S.K.A. is supported by DST/INSPIRE Research Grant No. IFA-PH-12, Department of Science and Technology, India. We thank Amol Dighe, Pankaj Agrawal, Ajit Srivastava, and Tarak Thakore for useful discussions. 


\section{A Oscillation of DM induced neutrinos}

The oscillation probability of neutrino from one flavor $(\alpha)$ to another flavor $(\beta)$ in vacuum is given by

$$
\begin{aligned}
P_{\alpha \beta}= & \sum_{k=l=1}^{3}\left|U_{\alpha k}\right|^{2}\left|U_{\beta l}\right|^{2} \\
& +2 \sum_{l>k} \operatorname{Re}\left(U_{\alpha k} U_{\beta k}^{*} U_{\alpha l}^{*} U_{\beta l}\right) \cos \left(\Delta E_{l k} L\right) \\
& -2 \sum_{l>k} \operatorname{Re}\left(U_{\alpha k} U_{\beta k}^{*} U_{\alpha l}^{*} U_{\beta l}\right) \sin \left(\Delta E_{l k} L\right),
\end{aligned}
$$

where $\mathrm{U}$ as the $3 \times 3$ unitary PMNS matrix [85-87]. When $L$ is very large, 2nd and 3rd terms in eq. (A.1) get averaged out to zero due to very rapid oscillations, and give rise to the following expression

$$
P_{\alpha \beta}=\sum_{k=1}^{3}\left|U_{\alpha k}\right|^{2}\left|U_{\beta k}\right|^{2} .
$$

We assume that the annihilation/decay of dark matter particles produce $\nu_{e}, \nu_{\mu}$, and $\nu_{\tau}$ in the ratio of 1:1:1 at the source. During their propagation through the astronomical distance from source to detector, they undergo vacuum oscillation. Imposing the unitary property of $U$ in eq. (A.2), the ratio of neutrino flavors at the Earth surface remains 1:1:1, and this is true irrespective of the values of oscillation parameters.

Open Access. This article is distributed under the terms of the Creative Commons Attribution License (CC-BY 4.0), which permits any use, distribution and reproduction in any medium, provided the original author(s) and source are credited.

\section{References}

[1] Planck collaboration, P.A.R. Ade et al., Planck 2015 results XIII. Cosmological parameters, Astron. Astrophys. 594 (2016) A13 [arXiv:1502.01589] [INSPIRE].

[2] F. Zwicky, Die Rotverschiebung von extragalaktischen Nebeln (in German), Helv. Phys. Acta 6 (1933) 110 [Gen. Relativ. Grav. 41 (2009) 207] [InSPIRE].

[3] V.C. Rubin and W.K. Ford, Jr., Rotation of the Andromeda nebula from a spectroscopic survey of emission regions, Astrophys. J. 159 (1970) 379 [INSPIRE].

[4] L.E. Strigari, Galactic searches for dark matter, Phys. Rept. 531 (2013) 1 [arXiv:1211.7090] [INSPIRE].

[5] D. Clowe et al., A direct empirical proof of the existence of dark matter, Astrophys. J. 648 (2006) L109 [astro-ph/0608407] [INSPIRE].

[6] WMaP Science Team collaboration, E. Komatsu et al., Results from the Wilkinson Microwave Anisotropy Probe, Prog. Theor. Exp. Phys. 2014 (2014) 06B102 [arXiv: 1404.5415] [INSPIRE].

[7] G. Steigman, Neutrinos and big bang nucleosynthesis, Adv. High Energy Phys. 2012 (2012) 268321 [arXiv: 1208.0032] [INSPIRE]. 
[8] G. Jungman, M. Kamionkowski and K. Griest, Supersymmetric dark matter, Phys. Rept. 267 (1996) 195 [hep-ph/9506380] [INSPIRE].

[9] G. Bertone, D. Hooper and J. Silk, Particle dark matter: evidence, candidates and constraints, Phys. Rept. 405 (2005) 279 [hep-ph/0404175] [INSPIRE].

[10] L. Bergström, Nonbaryonic dark matter: observational evidence and detection methods, Rept. Prog. Phys. 63 (2000) 793 [hep-ph/0002126] [inSPIRE].

[11] J. Ellis and K.A. Olive, Supersymmetric dark matter candidates, arXiv:1001.3651 [INSPIRE].

[12] DAMA, LIBRA collaboration, R. Bernabei et al., New results from DAMA/LIBRA, Eur. Phys. J. C 67 (2010) 39 [arXiv:1002.1028] [INSPIRE].

[13] LUX collaboration, D.S. Akerib et al., Improved limits on scattering of weakly interacting massive particles from reanalysis of 2013 LUX data, Phys. Rev. Lett. 116 (2016) 161301 [arXiv: 1512.03506] [INSPIRE].

[14] SuperCDMS collaboration, R. Agnese et al., New results from the search for low-mass weakly interacting massive particles with the CDMS low ionization threshold experiment, Phys. Rev. Lett. 116 (2016) 071301 [arXiv: 1509.02448] [INSPIRE].

[15] XENON100 collaboration, E. Aprile et al., Dark matter results from 225 live days of XENON100 data, Phys. Rev. Lett. 109 (2012) 181301 [arXiv:1207.5988] [INSPIRE].

[16] DARKSide collaboration, P. Agnes et al., Results from the first use of low radioactivity argon in a dark matter search, Phys. Rev. D 93 (2016) 081101 [arXiv:1510.00702] [INSPIRE].

[17] PandaX collaboration, X. Xiao et al., Low-mass dark matter search results from full exposure of the PandaX-I experiment, Phys. Rev. D 92 (2015) 052004 [arXiv:1505.00771] [INSPIRE].

[18] M. Lindner, A. Merle and V. Niro, Enhancing dark matter annihilation into neutrinos, Phys. Rev. D 82 (2010) 123529 [arXiv:1005.3116] [InSPIRE].

[19] S.K. Agarwalla, M. Blennow, E. Fernandez Martinez and O. Mena, Neutrino probes of the nature of light dark matter, JCAP 09 (2011) 004 [arXiv:1105.4077] [INSPIRE].

[20] Y. Farzan, Flavoring monochromatic neutrino flux from dark matter annihilation, JHEP 02 (2012) 091 [arXiv:1111.1063] [INSPIRE].

[21] P. Mijakowski, Direct and indirect search for dark matter, Ph.D. thesis, Inst. Nucl. Studies, Warsaw Poland, (2011) [INSPIRE].

[22] M. Blennow, M. Carrigan and E. Fernandez Martinez, Probing the dark matter mass and nature with neutrinos, JCAP 06 (2013) 038 [arXiv: 1303.4530] [INSPIRE].

[23] M. Gustafsson, T. Hambye and T. Scarna, Effective theory of dark matter decay into monochromatic photons and its implications: constraints from associated cosmic-ray emission, Phys. Lett. B 724 (2013) 288 [arXiv:1303.4423] [INSPIRE].

[24] C. El Aisati, M. Gustafsson, T. Hambye and T. Scarna, Dark matter decay to a photon and a neutrino: the double monochromatic smoking gun scenario, Phys. Rev. D 93 (2016) 043535 [arXiv: 1510.05008] [INSPIRE].

[25] L.A. Anchordoqui et al., IceCube neutrinos, decaying dark matter and the Hubble constant, Phys. Rev. D 92 (2015) 061301 [arXiv:1506.08788] [InSPIRE]. 
[26] C. Arina, S. Kulkarni and J. Silk, Monochromatic neutrino lines from sneutrino dark matter, Phys. Rev. D 92 (2015) 083519 [arXiv: 1506.08202] [INSPIRE].

[27] V. González-Macías and J. Wudka, Effective theories for dark matter interactions and the neutrino portal paradigm, JHEP 07 (2015) 161 [arXiv:1506.03825] [INSPIRE].

[28] V. González-Macías, J.I. Illana and J. Wudka, A realistic model for dark matter interactions in the neutrino portal paradigm, JHEP 05 (2016) 171 [arXiv:1601.05051] [INSPIRE].

[29] J.D. Zornoza, Indirect search for dark matter with neutrino telescopes, arXiv:1601.05691 [INSPIRE].

[30] C. Garcia-Cely and J. Heeck, Neutrino lines from Majoron dark matter, JHEP 05 (2017) 102 [arXiv: 1701.07209] [INSPIRE].

[31] J.F. Beacom, N.F. Bell and G.D. Mack, General upper bound on the dark matter total annihilation cross section, Phys. Rev. Lett. 99 (2007) 231301 [astro-ph/0608090] [INSPIRE].

[32] H. Yuksel, S. Horiuchi, J.F. Beacom and S. Ando, Neutrino constraints on the dark matter total annihilation cross section, Phys. Rev. D 76 (2007) 123506 [arXiv:0707.0196] [INSPIRE].

[33] Fermi-LAT collaboration, M. Ackermann et al., Updated search for spectral lines from galactic dark matter interactions with pass 8 data from the Fermi Large Area Telescope, Phys. Rev. D 91 (2015) 122002 [arXiv:1506.00013] [inSPIRE].

[34] R. Laha, K.C.Y. Ng, B. Dasgupta and S. Horiuchi, Galactic center radio constraints on gamma-ray lines from dark matter annihilation, Phys. Rev. D 87 (2013) 043516 [arXiv: 1208.5488] [INSPIRE].

[35] CMS collaboration, Search for dark matter and unparticles produced in association with a $Z$ boson in proton-proton collisions at $\sqrt{s}=8$ TeV, Phys. Rev. D 93 (2016) 052011 [arXiv: 1511.09375] [INSPIRE].

[36] CMS collaboration, Search for dark matter and large extra dimensions in pp collisions yielding a photon and missing transverse energy, Phys. Rev. Lett. 108 (2012) 261803 [arXiv:1204.0821] [INSPIRE].

[37] ATLAS collaboration, Search for dark matter candidates and large extra dimensions in events with a photon and missing transverse momentum in pp collision data at $\sqrt{s}=7 \mathrm{TeV}$ with the ATLAS detector, Phys. Rev. Lett. 110 (2013) 011802 [arXiv:1209.4625] [InSPIRE].

[38] A. Ghosh, T. Thakore and S. Choubey, Determining the neutrino mass hierarchy with INO,

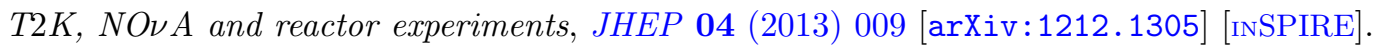

[39] M.M. Devi, T. Thakore, S.K. Agarwalla and A. Dighe, Enhancing sensitivity to neutrino parameters at INO combining muon and hadron information, JHEP 10 (2014) 189 [arXiv: 1406.3689] [INSPIRE].

[40] ICAL collaboration, S. Ahmed et al., Physics potential of the ICAL detector at the India-based Neutrino Observatory (INO), Pramana 88 (2017) 79 [arXiv:1505.07380] [INSPIRE].

[41] L.S. Mohan and D. Indumathi, Pinning down neutrino oscillation parameters in the 2-3 sector with a magnetised atmospheric neutrino detector: a new study, Eur. Phys. J. C 77 (2017) 54 [arXiv:1605. 04185] [INSPIRE]. 
[42] T. Thakore, A. Ghosh, S. Choubey and A. Dighe, The reach of INO for atmospheric neutrino oscillation parameters, JHEP 05 (2013) 058 [arXiv: 1303.2534] [INSPIRE].

[43] D. Kaur, M. Naimuddin and S. Kumar, The sensitivity of the ICAL detector at India-based Neutrino Observatory to neutrino oscillation parameters, Eur. Phys. J. C 75 (2015) 156 [arXiv: 1409.2231] [INSPIRE].

[44] N. Dash, V.M. Datar and G. Majumder, Sensitivity of the INO-ICAL detector to magnetic monopoles, Astropart. Phys. 70 (2015) 33 [arXiv: 1406.3938] [INSPIRE].

[45] A. Chatterjee, R. Gandhi and J. Singh, Probing Lorentz and CPT violation in a magnetized iron detector using atmospheric neutrinos, JHEP 06 (2014) 045 [arXiv:1402.6265] [INSPIRE].

[46] A. Chatterjee, P. Mehta, D. Choudhury and R. Gandhi, Testing nonstandard neutrino matter interactions in atmospheric neutrino propagation, Phys. Rev. D 93 (2016) 093017 [arXiv: 1409.8472] [INSPIRE].

[47] S. Choubey, A. Ghosh, T. Ohlsson and D. Tiwari, Neutrino physics with non-standard interactions at INO, JHEP 12 (2015) 126 [arXiv:1507.02211] [INSPIRE].

[48] S.P. Behera, A. Ghosh, S. Choubey, V.M. Datar, D.K. Mishra and A.K. Mohanty, Search for the sterile neutrino mixing with the ICAL detector at INO, Eur. Phys. J. C 77 (2017) 307 [arXiv: 1605.08607] [INSPIRE].

[49] R. de Grijs and G. Bono, Clustering of local group distances: publication bias or correlated measurements? IV. The galactic center, Astrophys. J. Suppl. 227 (2016) 5 [arXiv: 1610.02457].

[50] J.F. Navarro, C.S. Frenk and S.D.M. White, The structure of cold dark matter halos, Astrophys. J. 462 (1996) 563 [astro-ph/9508025] [INSPIRE].

[51] J. Diemand, M. Kuhlen and P. Madau, Dark matter substructure and gamma-ray annihilation in the milky way halo, Astrophys. J. 657 (2007) 262 [astro-ph/0611370] [INSPIRE].

[52] J. Stadel et al., Quantifying the heart of darkness with GHALO - a multi-billion particle simulation of our galactic halo, Mon. Not. Roy. Astron. Soc. 398 (2009) L21 [arXiv:0808.2981] [INSPIRE].

[53] J.F. Navarro et al., The diversity and similarity of cold dark matter halos, Mon. Not. Roy. Astron. Soc. 402 (2010) 21 [arXiv:0810.1522] [INSPIRE].

[54] G. Stinson, C. Brook, A.V. Maccio, J. Wadsley, T.R. Quinn and H.M.P. Couchman, Making galaxies in a cosmological context: the need for early stellar feedback, Mon. Not. Roy. Astron. Soc. 428 (2013) 129 [arXiv:1208.0002] [INSPIRE].

[55] A. Di Cintio et al., The dependence of dark matter profiles on the stellar-to-halo mass ratio: a prediction for cusps versus cores, Mon. Not. Roy. Astron. Soc. 437 (2014) 415 [arXiv: 1306.0898] [INSPIRE].

[56] E. Tollet et al., NIHAO - IV: core creation and destruction in dark matter density profiles across cosmic time, Mon. Not. Roy. Astron. Soc. 456 (2016) 3542 [arXiv:1507.03590] [INSPIRE].

[57] T.K. Chan et al., The impact of baryonic physics on the structure of dark matter haloes: the view from the FIRE cosmological simulations, Mon. Not. Roy. Astron. Soc. 454 (2015) 2981 [arXiv: 1507.02282] [INSPIRE]. 
[58] F. Marinacci, R. Pakmor and V. Springel, The formation of disc galaxies in high resolution moving-mesh cosmological simulations, Mon. Not. Roy. Astron. Soc. 437 (2014) 1750 [arXiv: 1305.5360] [INSPIRE].

[59] AGORA collaboration, J.-H. Kim et al., The AGORA high-resolution galaxy simulations comparison project, Astrophys. J. Suppl. 210 (2013) 14 [arXiv:1308.2669] [INSPIRE].

[60] J. Schaye et al., The EAGLE project: simulating the evolution and assembly of galaxies and their environments, Mon. Not. Roy. Astron. Soc. 446 (2015) 521 [arXiv:1407.7040] [INSPIRE].

[61] M. Schaller et al., Baryon effects on the internal structure of $\Lambda$ CDM haloes in the EAGLE simulations, Mon. Not. Roy. Astron. Soc. 451 (2015) 1247 [arXiv: 1409.8617] [InSPIRE].

[62] T. Sawala et al., The APOSTLE simulations: solutions to the local group's cosmic puzzles, Mon. Not. Roy. Astron. Soc. 457 (2016) 1931 [arXiv: 1511.01098] [INSPIRE].

[63] D.G. Cerdeno, M. Fornasa, A.M. Green and M. Peiro, How to calculate dark matter direct detection exclusion limits that are consistent with gamma rays from annihilation in the milky way halo, Phys. Rev. D 94 (2016) 043516 [arXiv: 1605.05185] [INSPIRE].

[64] A. Burkert and J. Silk, On the structure and nature of dark matter halos, in Dark matter in astrophysics and particle physics, H.V. Klapdor-Kleingrothaus and L. Baudis eds., (1999), pg. 375 [astro-ph/9904159] [INSPIRE].

[65] ICECube collaboration, M.G. Aartsen et al., Search for dark matter annihilation in the galactic center with IceCube-79, Eur. Phys. J. C 75 (2015) 492 [arXiv:1505.07259] [INSPIRE].

[66] S. Ando, Can dark matter annihilation dominate the extragalactic gamma-ray background?, Phys. Rev. Lett. 94 (2005) 171303 [astro-ph/0503006] [INSPIRE].

[67] K.C.Y. Ng et al., Resolving small-scale dark matter structures using multisource indirect detection, Phys. Rev. D 89 (2014) 083001 [arXiv:1310.1915] [InSPIRE].

[68] S. Campbell, Gamma-ray probes of dark matter substructure, AIP Conf. Proc. 1604 (2014) 11 [INSPIRE].

[69] C.A. Correa, J.S.B. Wyithe, J. Schaye and A.R. Duffy, The accretion history of dark matter haloes - III. A physical model for the concentration-mass relation, Mon. Not. Roy. Astron. Soc. 452 (2015) 1217 [arXiv: 1502.00391] [INSPIRE].

[70] R. Bartels and S. Ando, Boosting the annihilation boost: tidal effects on dark matter subhalos and consistent luminosity modeling, Phys. Rev. D 92 (2015) 123508 [arXiv: 1507.08656] [INSPIRE].

[71] Á. Moliné, M.A. Sánchez-Conde, S. Palomares-Ruiz and F. Prada, Characterization of subhalo structural properties and implications for dark matter annihilation signals, Mon. Not. Roy. Astron. Soc. 466 (2017) 4974 [arXiv: 1603.04057] [INSPIRE].

[72] S. Palomares-Ruiz, Model-independent bound on the dark matter lifetime, Phys. Lett. B 665 (2008) 50 [arXiv:0712.1937] [INSPIRE].

[73] India-based Neutrino Observatory (INO) webpage, http://www.ino.tifr.res.in/ino/.

[74] S.P. Behera, M.S. Bhatia, V.M. Datar and A.K. Mohanty, Simulation studies for electromagnetic design of INO ICAL magnet and its response to muons, arXiv:1406.3965 [INSPIRE]. 
[75] A. Chatterjee et al., A simulations study of the muon response of the iron calorimeter detector at the India-based Neutrino Observatory, 2014 JINST 9 P07001 [arXiv:1405.7243] [INSPIRE].

[76] M.M. Devi et al., Hadron energy response of the iron calorimeter detector at the India-based Neutrino Observatory, 2013 JINST 8 P11003 [arXiv:1304.5115] [INSPIRE].

[77] L.S. Mohan et al., Simulation studies of hadron energy resolution as a function of iron plate thickness at INO-ICAL, 2014 JINST 9 T09003 [arXiv:1401.2779] [INSPIRE].

[78] S.T. Petcov and T. Schwetz, Determining the neutrino mass hierarchy with atmospheric neutrinos, Nucl. Phys. B 740 (2006) 1 [hep-ph/0511277] [INSPIRE].

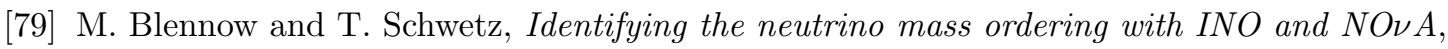
JHEP 08 (2012) 058 [Erratum ibid. 11 (2012) 098] [arXiv:1203.3388] [INSPIRE].

[80] M. Ghosh, P. Ghoshal, S. Goswami and S.K. Raut, Can atmospheric neutrino experiments provide the first hint of leptonic CP-violation?, Phys. Rev. D 89 (2014) 011301 [arXiv:1306.2500] [INSPIRE].

[81] M. Ghosh, P. Ghoshal, S. Goswami and S.K. Raut, Evidence for leptonic CP phase from

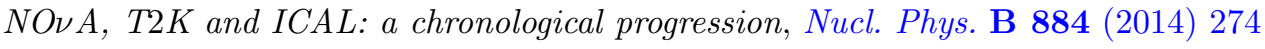
[arXiv: 1401.7243] [INSPIRE].

[82] R. Gandhi, P. Ghoshal, S. Goswami, P. Mehta, S.U. Sankar and S. Shalgar, Mass hierarchy determination via future atmospheric neutrino detectors, Phys. Rev. D 76 (2007) 073012 [arXiv:0707.1723] [INSPIRE].

[83] J.A. Formaggio and G.P. Zeller, From eV to EeV: neutrino cross sections across energy scales, Rev. Mod. Phys. 84 (2012) 1307 [arXiv:1305.7513] [INSPIRE].

[84] M. Honda, M. Sajjad Athar, T. Kajita, K. Kasahara and S. Midorikawa, Atmospheric neutrino flux calculation using the NRLMSISE-00 atmospheric model, Phys. Rev. D 92 (2015) 023004 [arXiv: 1502.03916] [INSPIRE].

[85] B. Pontecorvo, Inverse beta processes and nonconservation of lepton charge, Sov. Phys. JETP 7 (1958) 172 [Zh. Eksp. Teor. Fiz. 34 (1957) 247] [InSPIRE].

[86] Z. Maki, M. Nakagawa and S. Sakata, Remarks on the unified model of elementary particles, Prog. Theor. Phys. 28 (1962) 870 [InSPIRE].

[87] B. Pontecorvo, Neutrino experiments and the problem of conservation of leptonic charge, Sov. Phys. JETP 26 (1968) 984 [Zh. Eksp. Teor. Fiz. 53 (1967) 1717] [InSPIRE].

[88] A. Dziewonski and D. Anderson, Preliminary reference earth model, Phys. Earth Planet. Interiors 25 (1981) 297 [INSPIRE].

[89] D.V. Forero, M. Tortola and J.W.F. Valle, Neutrino oscillations refitted, Phys. Rev. D 90 (2014) 093006 [arXiv: 1405.7540] [INSPIRE].

[90] I. Esteban, M.C. Gonzalez-Garcia, M. Maltoni, I. Martinez-Soler and T. Schwetz, Updated fit to three neutrino mixing: exploring the accelerator-reactor complementarity, JHEP 01 (2017) 087 [arXiv:1611.01514] [INSPIRE].

[91] F. Capozzi, E. Di Valentino, E. Lisi, A. Marrone, A. Melchiorri and A. Palazzo, Global constraints on absolute neutrino masses and their ordering, Phys. Rev. D 95 (2017) 096014 [arXiv: 1703.04471] [INSPIRE]. 
[92] H. Nunokawa, S.J. Parke and R. Zukanovich Funchal, Another possible way to determine the neutrino mass hierarchy, Phys. Rev. D 72 (2005) 013009 [hep-ph/0503283] [INSPIRE].

[93] A. de Gouvêa, J. Jenkins and B. Kayser, Neutrino mass hierarchy, vacuum oscillations and vanishing $\left|U_{e 3}\right|$, Phys. Rev. D 71 (2005) 113009 [hep-ph/0503079] [INSPIRE].

[94] Particle Data Group collaboration, C. Patrignani et al., Review of particle physics, Chin. Phys. C 40 (2016) 100001 [inSPIRE].

[95] M. Honda, T. Kajita, K. Kasahara, S. Midorikawa and T. Sanuki, Calculation of atmospheric neutrino flux using the interaction model calibrated with atmospheric muon data, Phys. Rev. D 75 (2007) 043006 [astro-ph/0611418] [INSPIRE].

[96] P. Huber, M. Lindner and W. Winter, Superbeams versus neutrino factories, Nucl. Phys. B 645 (2002) 3 [hep-ph/0204352] [INSPIRE].

[97] G.L. Fogli, E. Lisi, A. Marrone, D. Montanino, A. Palazzo and A.M. Rotunno, Solar neutrino oscillation parameters after first KamLAND results, Phys. Rev. D 67 (2003) 073002 [hep-ph/0212127] [INSPIRE].

[98] M.C. Gonzalez-Garcia and M. Maltoni, Atmospheric neutrino oscillations and new physics, Phys. Rev. D 70 (2004) 033010 [hep-ph/0404085] [INSPIRE].

[99] S.P. Mikheev and A. Yu. Smirnov, Resonance amplification of oscillations in matter and spectroscopy of solar neutrinos, Sov. J. Nucl. Phys. 42 (1985) 913 [Yad. Fiz. 42 (1985) 1441] [INSPIRE].

[100] S.P. Mikheev and A. Yu. Smirnov, Resonant amplification of neutrino oscillations in matter and solar neutrino spectroscopy, Nuovo Cim. C 9 (1986) 17 [INSPIRE].

[101] L. Wolfenstein, Neutrino oscillations in matter, Phys. Rev. D 17 (1978) 2369 [INSPIRE].

[102] L. Wolfenstein, Neutrino oscillations and stellar collapse, Phys. Rev. D 20 (1979) 2634 [INSPIRE].

[103] N. Dash, V.M. Datar and G. Majumder, Sensitivity for detection of decay of dark matter particle using ICAL at INO, Pramana 86 (2016) 927 [arXiv:1410.5182] [INSPIRE].

[104] Super-Kamiokande collaboration, P. Mijakowski, Indirect searches for dark matter particles at Super-Kamiokande, J. Phys. Conf. Ser. 718 (2016) 042040 [InSPIRE].

[105] ICECuBe collaboration, M.G. Aartsen et al., Search for neutrinos from dark matter self-annihilations in the center of the milky way with 3 years of IceCube/DeepCore, arXiv: 1705.08103 [INSPIRE].

[106] ANTARES collaboration, S. Adrian-Martinez et al., Search of dark matter annihilation in the galactic centre using the ANTARES neutrino telescope, JCAP 10 (2015) 068 [arXiv: 1505. 04866] [INSPIRE].

[107] A. Albert et al., Results from the search for dark matter in the milky way with 9 years of data of the ANTARES neutrino telescope, Phys. Lett. B 769 (2017) 249 [arXiv: 1612.04595] [INSPIRE].

[108] IceCube PINGU collaboration, M.G. Aartsen et al., Letter of intent: the Precision IceCube Next Generation Upgrade (PINGU), arXiv:1401.2046 [INSPIRE].

[109] A.D. Avrorin et al., Dark matter constraints from an observation of dSphs and the LMC with the Baikal NT200, arXiv:1612.03836 [INSPIRE]. 
[110] ICECUBE collaboration, R. Abbasi et al., Search for dark matter from the galactic halo with the IceCube neutrino telescope, Phys. Rev. D 84 (2011) 022004 [arXiv:1101.3349] [INSPIRE].

[111] B. Dasgupta and R. Laha, Neutrinos in IceCube/KM3NeT as probes of dark matter substructures in galaxy clusters, Phys. Rev. D 86 (2012) 093001 [arXiv:1206.1322] [INSPIRE].

[112] ICECuBe collaboration, M.G. Aartsen et al., IceCube search for dark matter annihilation in nearby galaxies and galaxy clusters, Phys. Rev. D 88 (2013) 122001 [arXiv:1307.3473] [INSPIRE].

[113] IceCube collaboration, M.G. Aartsen et al., Multipole analysis of IceCube data to search for dark matter accumulated in the galactic halo, Eur. Phys. J. C 75 (2015) 20 [arXiv: 1406.6868] [INSPIRE].

[114] Á. Moliné, A. Ibarra and S. Palomares-Ruiz, Future sensitivity of neutrino telescopes to dark matter annihilations from the cosmic diffuse neutrino signal, JCAP 06 (2015) 005 [arXiv: 1412.4308] [INSPIRE].

[115] C. Rott, K. Kohri and S.C. Park, Superheavy dark matter and IceCube neutrino signals: bounds on decaying dark matter, Phys. Rev. D 92 (2015) 023529 [arXiv:1408.4575] [INSPIRE].

[116] C. El Aisati, M. Gustafsson and T. Hambye, New search for monochromatic neutrinos from dark matter decay, Phys. Rev. D 92 (2015) 123515 [arXiv:1506.02657] [INSPIRE].

[117] M. Chianese, G. Miele, S. Morisi and E. Vitagliano, Low energy IceCube data and a possible dark matter related excess, Phys. Lett. B 757 (2016) 251 [arXiv: 1601.02934] [INSPIRE].

[118] S.M. Boucenna et al., Decaying leptophilic dark matter at IceCube, JCAP 12 (2015) 055 [arXiv: 1507.01000] [INSPIRE].

[119] ICECuBE collaboration, M.G. Aartsen et al., All-flavour search for neutrinos from dark matter annihilations in the milky way with IceCube/DeepCore, Eur. Phys. J. C 76 (2016) 531 [arXiv: 1606.00209] [INSPIRE].

[120] J. Kumar and P. Sandick, Searching for dark matter annihilation to monoenergetic neutrinos with liquid scintillation detectors, JCAP 06 (2015) 035 [arXiv:1502.02091] [INSPIRE].

[121] LENA collaboration, M. Wurm et al., The next-generation liquid-scintillator neutrino observatory LENA, Astropart. Phys. 35 (2012) 685 [arXiv:1104.5620] [INSPIRE].

[122] M. Kachelriess and P.D. Serpico, Model-independent dark matter annihilation bound from the diffuse $\gamma$ ray flux, Phys. Rev. D 76 (2007) 063516 [arXiv: 0707.0209] [InSPIRE].

[123] N.F. Bell, J.B. Dent, T.D. Jacques and T.J. Weiler, Electroweak bremsstrahlung in dark matter annihilation, Phys. Rev. D 78 (2008) 083540 [arXiv:0805.3423] [INSPIRE].

[124] N.F. Bell, J.B. Dent, T.D. Jacques and T.J. Weiler, Dark matter annihilation signatures from electroweak bremsstrahlung, Phys. Rev. D 84 (2011) 103517 [arXiv:1101.3357] [INSPIRE].

[125] N.F. Bell, J.B. Dent, A.J. Galea, T.D. Jacques, L.M. Krauss and T.J. Weiler, $W / Z$ bremsstrahlung as the dominant annihilation channel for dark matter, revisited, Phys. Lett. B 706 (2011) 6 [arXiv:1104.3823] [INSPIRE]. 
[126] M. Cirelli et al., PPPC 4 DM ID: a Poor Particle Physicist Cookbook for Dark Matter Indirect Detection, JCAP 03 (2011) 051 [Erratum ibid. 10 (2012) E01] [arXiv:1012.4515] [INSPIRE].

[127] K. Murase, R. Laha, S. Ando and M. Ahlers, Testing the dark matter scenario for PeV neutrinos observed in IceCube, Phys. Rev. Lett. 115 (2015) 071301 [arXiv:1503.04663] [INSPIRE].

[128] A. Esmaili and P.D. Serpico, Gamma-ray bounds from EAS detectors and heavy decaying dark matter constraints, JCAP 10 (2015) 014 [arXiv: 1505.06486] [INSPIRE].

[129] D. Chowdhury, A.M. Iyer and R. Laha, Constraints on dark matter annihilation to fermions and a photon, arXiv:1601.06140 [INSPIRE].

[130] F.S. Queiroz, C.E. Yaguna and C. Weniger, Gamma-ray limits on neutrino lines, JCAP 05 (2016) 050 [arXiv: 1602.05966] [INSPIRE].

[131] A.D. Avrorin et al., Sensitivity of the Baikal-GVD neutrino telescope to neutrino emission toward the center of the galactic dark matter halo, JETP Lett. 101 (2015) 289 [arXiv: 1412.3672] [INSPIRE].

[132] K. Abe et al., Letter of intent: the Hyper-Kamiokande experiment - detector design and physics potential, arXiv:1109.3262 [INSPIRE].

[133] E.G. Speckhard, K.C.Y. Ng, J.F. Beacom and R. Laha, Dark matter velocity spectroscopy, Phys. Rev. Lett. 116 (2016) 031301 [arXiv: 1507. 04744] [INSPIRE].

[134] D. Powell, R. Laha, K.C.Y. Ng and T. Abel, Doppler effect on indirect detection of dark matter using dark matter only simulations, Phys. Rev. D 95 (2017) 063012 [arXiv: 1611.02714] [INSPIRE]. 